\title{
Meinungswandel und Bedeutungswandel.
}

\section{Ein Problem der Analyse kulturellen Wandels, dargestellt an schulischen Zielwerten in der Bundesrepublik Deutschland zwischen 1958 und 1979}

\author{
Heiner Meulemann \\ Universität zu Köln, Zentralarchiv für empirische Sozialforschung, Bachemer Straße 40, D-5000 Köln 41
}

\begin{abstract}
Zusammenfassung: Der gemessene Wandel von Antworthäufigkeiten in Umfragen zu verschiedenen Zeitpunkten kann auf einen Meinungswandel und auf einen Bedeutungswandel hinweisen. Wenn für jeden Zeitpunkt mehrere theoretisch geordnete Fragen vorliegen, dann kann die Beziehungsstruktur als ein Indikator für die Bedeutung der Fragen angesehen und durch den Wandel der Beziehungsstruktur der Bedeutungswandel unabhängig vom gemessenen Wandel erfaßt werden. Bleibt die Beziehungsstruktur konstant, so kann der gemessene Wandel als Meinungswandel gewertet werden. Diese Forschungsstrategie wird an einem Inventar schulischer Zielwerte erprobt, das 1958, 1973 und 1979 erhoben wurde. Um Stabilität oder Wandel der Bedeutungen zu prüfen, werden Prokrustes-Rotationen der Konfigurationen aus multidimensionalen Skalierungen für jeden Zeitpunkt verwandt. Die Bedeutungen sind in den Gesamtstichproben durchgängig und in Teilstichproben gerade dort konstant, wo der stärkste gemessene Wandel auftritt. Der gemessene Wandel der schulischen Zielwerte kann also tatsächlich als Meinungswandel aufgefaßt werden.
\end{abstract}

\section{Messung des Wandels und Wandel der Messungen: Das Problem und ein Lösungsvorschlag}

Der kulturelle Wandel war, wie der strukturelle Wandel, ein Thema der Soziologie von Anfang an. Schon die Begründer der Soziologie - Saint Simon, Comte und Durkheim, Marx, Weber und Simmel beschäftigte die Frage, wie sich die Glaubenssysteme und Wertmuster in einer Gesellschaft entwikkeln, ebenso stark wie die Frage, wie die zum Überleben einer Gesellschaft notwendige Arbeit auf soziale Gruppen verteilt wird. Während aber für die Analyse des strukturellen Wandels die nationalen statistischen Ämter, die seit dem Ende des 19. Jahrhunderts gegründet wurden, eine solide und breite Datenbasis hervorbrachten, fehlte eine vergleichbare Datenbasis lange für die Analyse des kulturellen Wandels. Erst die auf repräsentativen Stichproben beruhende Meinungsforschung, die in ihren Anfängen in den Zwischenkriegsjahren der USA vor allem zur Lösung sozialer Probleme beitragen und politische Interessen unterstützen sollte (Boyd/Hyman 1975: 306-324), konnte auch zur Erhebung von Meinungen und Überzeugungen, Vorstellungen und Einstellungen, Normen und Werten in der breiten Bevölkerung genutzt werden. Und erst nach dem Zweiten Weltkrieg wurden derartige Befragungen häufig genug wiederholt, um einen längerfristigen Zeitvergleich und eine Analyse des kulturellen Wandels zuzulassen (Davis 1975; Duncan 1975; Allerbeck 1976).
Meinungsbefragungen bedienen sich des Mittels der Sprache. Selbst in einer standardisierten Befragung bleiben die Frageformulierungen und die Antwortvorgaben unvermeidlich in einem bestimmten Maße interpretationsfähig, vieldeutig. Das ist nicht sehr problematisch, solange Frage und Antwort auf einen klar umrissenen Sachverhalt zielen, solange etwa nach der gewählten Partei, der besuchten Schule oder dem Ferienziel des letzten Jahres gefragt wird; die Denotation ist hier eindeutig, so sehr die Konnotationen schwanken mögen. Das wird jedoch durchaus problematisch, sobald Frage und Antwort sich auf allein in der Sprache greifbare Sachverhalte beziehen, wie es bei Befragungen zu kulturellen Sachverhalten per definitionem der Fall ist. Die Werte und Normen, die als kulturelle Sachverhalte angesehen werden, liegen auf einer höheren Allgemeinheitsstufe als Verhaitensweisen oder Äußerungen; ohne feste Denotation können sie mit verschiedenen Konnotationen verbunden werden. „Selbstverwirklichung" kann als Meisterung von Ansprüchen der Umwelt in Beruf und Familie, aber auch als Befreiung des Selbst von äußeren Ansprüchen verstanden werden; „Ordnung und Disziplin“ kann als selbst auferlegtes Prinzip der Lebensführung, aber auch als autoritäre Fremdbestimmung gewertet werden. Wenn man nun Normen und Werte erfragt, so können Unterschiede zwischen Personen oder Gruppen entweder dadurch entstehen, daß Werte und Normen unterschiedlich verstanden werden, oder dadurch, daß sie unterschiedlich stark unterstützt werden. 
Das gleiche Problem findet sich bei der Analyse des kulturellen Wandels, in der nicht mehr Unterschiede zwischen Gruppen zu einem Zeitpunkt, sondern Gesamtgruppen zu verschiedenen Zeitpunkten betrachtet werden. Wenn der Anteil der Bevölkerung, der einen bestimmten Wert bejaht, mit der Zeit steigt oder fällt, so kann es sein, daß die Bedeutung des Wertes sich verschoben hat oder daß die Bevölkerung tatsächlich in verstärktem oder abgeschwächtem Maße sich diesen Wert zu eigen gemacht hat. Wenn der Anteil der Bevölkerung, der einen bestimmten Wert bejaht, konstant bleibt, so kann es sein, daß die Meinungen und Bedeutungen sich gegenläufig entwickelt und neutralisiert haben. Hat sich die Realität oder das Instrument gewandelt? Wird Wandel gemessen oder wandeln sich die Messungen? Stellt der gemessene Wandel einen Meinungswandel oder einen Bedeutungswandel dar? Im folgenden soll eine Strategie, wie diese Frage empirisch behandelt werden kann, entwickelt und an einem Beispiel erprobt werden.

Die Analyse kulturellen Wandels mit Umfragen setzt voraus, daß mit gleichen Frageformulierungen gearbeitet wird. Die Beschreibung auch nur des gemessenen Wandels ist ohne gleiche Formulierung unmöglich (Schumann/Presser 1981: 312; Sudmann/Bradburn 1982: 121). Sprachliche Äußerungen sind zwar interpretationsfähig, aber über Interpretationen kann nur gestritten werden, wenn man sich auf verbindliche Bedeutungen stützen kann. Das gilt für zwanglose Alltagssituationen ebenso wie für die hoch typisierte Situation des Interviews. Gleiche Formulierungen garantieren zwar keine gleichen Bedeutungen, aber über unterschiedliche Bedeutungen läßt sich nur an gleichen Formulierungen urteilen. Die wörtliche Gleichheit der Formulierungen von Fragen und Antwortvorgaben ist Voraussetzung für die Analyse des kulturellen Wandels, nicht weil sie Gleichheit der Bedeutungen garantiert, sondern weil sie überhaupt erst einen Maßstab liefert, an dem sich mögliche Bedeutungsänderungen messen lassen. ${ }^{1}$

Hypothesen über einen möglichen Bedeutungswandel lassen sich nun am einfachsten aus der Beschreibung des gleichzeitigen Meinungswandels gewinnen. ${ }^{2}$ Wann immer der Anteil einer Bevölkerungsgruppe, der einen bestimmten Wert unterstützt, sich deutlich wandelt, sollte man überlegen, ob nicht ein spezifischer Bedeutungswandel dahinter gestanden haben kann. Erst wenn Hypothesen über einen Bedeutungswandel empirisch ausge- schlossen worden sind, kann man den gemessenen Wandel als einen Meinungswandel interpretieren. Wie aber lassen sich nun Meinungswandel und Bedeutungswandel am gemessenen Wandel empirisch trennen? Hier ist die Zahl der Indikatoren entscheidend, mit denen der Wandel gemessen wurde.

Solange man nur über einen Indikator im Zeitablauf verfügt, können Meinungs- und Bedeutungswandel nicht getrennt werden. Die Bedeutung kann zwar aus dem Wortlaut des Indikators expliziert, ein mutmaßlicher Bedeutungswandel aber empirisch nicht unabhängig vom gemessenen Wandel erfaßt werden. Sobald man jedoch über mehrere Indikatoren verfügt, kann die Bedeutung dieser Indikatoren als die Struktur ihrer Beziehungen verstanden werden. Die Bedeutung jedes einzelnen Indikators ist zwar nach wie vor nur durch eine semantische Explikation und nicht durch empiri-

' Die Forderung nach wörtlicher Gleichheit der Frageformulierungen steht nicht im Widerspruch zu der Möglichkeit, daß wörtlich nicht gleiche Formulierungen äquivalent sein können. Denn diese Möglichkeit rechtfertigt nicht, beliebig von der Forderung abzuweichen, sondern nur dann, wenn die Äquivalenz des Ungleichen theoretisch genau bestimmt worden ist. Diese Probleme sind in der interkulturell vergleichenden Forschung unter der Überschrift „Identität vs. Äquivalenz" und „funktionelle Äquivalenz" ausführlich diskutiert worden (Przeworski/Teune 1970: 113-131; Nowak 1977: 34-45). - Selbst bei identischen Formulierungen kann aber u.U. der unterschiedliche Kontext der Fragen in den Fragebögen der Replikationsstudien unterschiedliche Bedeutungen suggerieren. Nach empirischen Untersuchungen ist dies vor allem dann der Fall, wenn relativ eng verwandte Fragen von Befragung zu Befragung in ihrer Abfolge variiert werden, weniger jedoch für größere Fragekomplexe (Schumann/Presser 1981: 23-77).

${ }^{2}$ Sicher ist der gleichzeitige strukturelle Wandel auch eine wichtige Quelle für Hypothesen über Bedeutungswandlungen. So könnten etwa Veränderungen in der Struktur der Erwerbstätigen (wie die Zunahme von Teilzeit- und Nebenerwerbstätigkeit) als Ursache für veränderte Bedeutungen des Wertes „Arbeit" in der Bevölkerung gesehen werden: ein gemessener Wandel des Wertes „Arbeit" könnte hypothetisch zunächst als strukturell veranlaßter Bedeutungswandel verstanden werden, ohne sofort als Meinungswandel (,Wertwan-

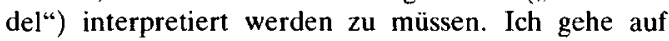
strukturelle Wandlungen nicht weiter ein, um mich auf die beiden Komponenten des gemessenen kulturellen Wandels, Meinungs- und Bedeutungswandel, beschränken zu können. 
sche Meßzahlen erfaßbar, aber die Indikatoren stehen zueinander in Beziehungen, die sich unabhängig vom Meinungswandel in der Zeit verändern können. Die Beziehungen der Indikatoren untereinander weisen auf Bedeutungsaspekte jedes einzelnen Indikators - so wie in der semantischen Theorie die Bedeutung eines Wortes durch die Bedeutungskomponenten bestimmt ist, die es mit anderen Worten teilt (Katz/Fodor 1963). Um Meinungs- und Bedeutungswandel zu trennen, müssen daher zu jedem Zeitpunkt zwei Indikatoren kreuztabelliert und der Wandel der zeitlichen Unterstützung jedes Indikators vom Wandel der Beziehung zwischen den beiden Indikatoren getrennt werden. Statistisch gesehen spitzt sich das Problem darauf zu, in einer Kreuztabelle Effekte der Randverteilung von Effekten der Zellenbesetzung zu trennen. Da dieses statistische Problem lösbar ist, können Meinungswandel und Bedeutungswandel getrennt werden.

Wenn man mehrere Indikatoren zur Betrachtung des Wandels heranzieht, sollte man die Beziehungsstruktur zwischen den Indikatoren a priori theoretisch bestimmen; denn die Struktur ist ein Mittel, um den Wandel zu erfassen. Die hypothetisch gesetzte Struktur der Beziehungen muß dann ebenfalls empirisch überprüft werden - getrennt für jeden Zeitpunkt. Die Überprüfung der Struktur für alle Zeitpunkte aber ist mit der Überprüfung des Bedeutungswandels identisch. Hypothesen über die Beziehungsstruktur der Indikatoren auf der einen Seite und Hypothesen über Bedeutungswandlungen auf der anderen Seite können zwar unabhängig voneinander entwickelt, aber nicht unabhängig voneinander überprüft werden: Wie in einem experimentum crucis sind zwei wi- dersprüchliche Voraussagen auf eine empirische Prüfung gerichtet. Hypothesen über die Beziehungsstruktur werden um so glaubwürdiger, je weniger, Hypothesen über den Wandel, je mehr die Struktur sich wandelt. Auch wenn hier beide Arten von Hypothesen nur gleichzeitig überprüft werden können, haben Hypothesen über die Struktur logisch Vorrang vor Hypothesen über den Bedeutungswandel. Deshalb werde ich beide Arten von Hypothesen ihrer logischen Rangfolge nach entwickeln.

Für die Analyse von Meinungs- und Bedeutungswandel schlage ich also folgende Strategie vor: Auf der einen Seite sollen aus dem gemessenen Wandel Hypothesen über den Bedeutungswandel entwikkelt werden, auf der anderen Seite sollen diese Hypothesen unabhängig vom gemessenen Wandel überprüft werden. Die unabhängige Überprüfung ist möglich, wenn mehrere Indikatoren für den Wandel zur Verfügung stehen, über deren Beziehungsstruktur Hypothesen vorgegeben sind. Die Hypothesen über die Struktur, zeitlich betrachtet, widersprechen dann direkt den Hypothesen über den Bedeutungswandel, und der Wandel der Beziehungsstruktur erfaßt den Bedeutungswandel. Wenn die Hypothesen über den Bedeutungswandel sich nicht bestätigen und die Beziehungsstruktur zeitlich konstant bleibt, dann kann der gemessene Wandel als ein Meinungswandel verstanden werden. Wenn die Hypothesen über den Bedeutungswandel sich bestätigen und die Indikatoren keine zeitlich konstante Struktur aufweisen, dann muß der gemessene Wandel als ein Produkt von Meinungs- und Bedeutungswandel angesehen und im einzelnen analysiert werden. Man kann diese Strategie in folgendem Schema veranschaulichen:

Interpretationen möglicher Kombinationen von Messung und Bedeutung

Bedeutung (Beziehungen)

konstant

variabel gemessener Wandel $=$ Meinungswandel variabel

gemessener Wandel $=$ Produkt von Meinungs- und Bedeutungswandel

Messung

(Verteilungen)

konstant gemessene Konstanz $=$ Meinungskonstanz gemessene Konstan $z=$ Produkt von Meinungs- und Bedeutungswandel 
Ich will im folgenden diese Strategie am Beispiel schulischer Zielwerte zwischen 1958 und 1979 erproben: Zuerst werde ich Hypothesen über die Beziehungsstruktur zwischen den Werten darstellen und aus dem gemessenem Wandel der Werte Hypothesen über den Bedeutungswandel entwikkeln (Abschnitt 2). Dann werde ich die Hypothesen über die Beziehungsstruktur und den Bedeutungswandel überprüfen - zuerst für die gesamte Bevölkerung (Abschnitt 3), dann für Teilgruppen, in denen man spezifische Bedeutungswandlungen vermuten kann (Abschnitt 4).

\section{Mutmaßliche Bedeutungsstruktur, gemessener Wandel und mutmaßlicher Bedeutungswandel}

Die schulischen Zielwerte wurden durch ein Inventar von 9 Vorgaben erhoben, aus denen die Befragten drei als die wichtigsten auswählen mußten. Die Zielwerte lassen sich theoretisch in zwei Dimensionen ordnen: dem Wertinhalt - kognitiv und evaluativ - und dem Abstraktionsgrad - niedrig und hoch. Kognitive Zielwerte lassen sich eher durch ein Training des Verstandes, evaluative Zielwerte eher durch ein Training des Willens erreichen; kognitive Zielwerte sind auf Wissen, evaluative Zielwerte auf Handeln gerichtet. Ein niedriger Abstraktionsgrad impliziert, daß die Zielerreichung leicht kontrolliert werden kann, durch Abfragen des Wissens oder durch Beobachtung des Handelns; ein hoher Abstraktionsgrad impliziert, daß die Zielerreichung nur indirekt kontrolliert werden kann, durch Schlüsse auf Prinzipien des Wissens oder auf Regeln des Handelns. Durch Kreuzung der Dimensionen Wertinhalt und Abstraktionsniveau ergeben sich vier Gruppen von Zielwerten.

Kognitive Zielwerte auf niedrigem Abstraktionsniveau bezeichne ich als Fertigkeiten: „Kenntnisse für den Beruf" und ,Vielseitiges Wissen"; kognitive Zielwerte auf hohem Abstraktionsniveau als Kompetenzen: „Eigene Urteilsfähigkeit“. Fertigkeiten sind als spezifische Inhalte oder für spezifische Zwecke gedacht, Kompetenzen beziehen sich auf die Prinzipien des Umgangs mit spezifischen Inhalten oder Zwecken. Evaluative Zielwerte auf niedrigem Abstraktionsniveau bezeichne ich als Werte der Konvention: „Ordnung und Disziplin“ und „Gute Umgangsformen"; evaluative Zielwerte auf hohem Abstraktionsniveau als AutonomieWerte: „Persönliche Selbständigkeit", „Sicheres Selbstbewußtsein“, „Achtung vor den Mitmen- schen“, „Lebensfreude“. Werte der Konvention beziehen sich auf sichtbares Verhalten, Autonomie-Werte auf verinnerlichte Haltungen, die erschlossen werden müssen.

Diese theoretische Ordnung der Zielwerte liefert nun die Hypothesen über die Struktur der Werte, die erste Art von Hypothesen dieser Analyse. Sie dient aber zugleich auch als Mittel zur Beschreibung des gemessenen Wandels, aus dem Hypothesen über den Bedeutungswandel, die zweite Art von Hypothesen dieser Analyse, gewonnen werden sollen. Der gemessene Wandel der schulischen Zielwerte von 1958, 1973 bis 1979, der an anderer Stelle (Meulemann 1982a: 232-235) ausführlich dargestellt wird, ist erstaunlich durchgängig; es gibt nur eine minimale Inversion der Richtung des Wandels. Inhaltlich läßt sich der gemessene Wandel in zwei Punkten zusammenfassen. Erstens gewinnen alle drei kognitiven Zielwerte auf Kosten der evaluativen Zielwerte. Zweitens aber ist der Wandel innerhalb der evaluativen Zielwerte differenziert: Während „Ordnung und Disziplin“, „Gute Umgangsformen“ und „Achtung vor den Mitmenschen" drastisch und „Lebensfreude" minimal zurückgehen, gewinnen „Persönliche Selbständigkeit“ und „Sicheres Selbstbewußtsein“ entgegen dem allgemeinen Rückgang evaluativer Zielwerte etwas an Boden. Nach dem Entwicklungsverlauf müßte "Achtung vor den Mitmenschen“" zu den Konventions-Werten und nicht, wie in der Systematik vorausgesetzt, zu den Autonomie-Werten gerechnet werden: „Ordnung und Disziplin“, „Gute Umgangsformen“ und „Achtung vor den Mitmenschen“ könnten dann nachträglich als kollektiv-orientierte Werte „Persönlicher Selbständigkeit" und „Sicherem Selbstbewußtsein" als selbstorientierten Werten (Parsons/Shils 1951: 76-91) gegenübergestellt werden. Die Dimension Konvention-Autonomie wird von der Dimension Kollektivorientierung-Selbstorientierung überlagert. „Ordnung und Disziplin“ und „Gute Umgangsformen" sind in konventioneller Weise auf Kollektive orientiert, „Sicheres Selbstbewußtsein“ und „Persönliche Selbständigkeit" in autonomer Form auf das Selbst orientiert; „Achtung vor den Mitmenschen" aber steht zwischen diesen beiden Gruppen: sie ist in autonomer Form auf Kollektive orientiert. Daß gleichzeitig „Achtung vor den Mitmenschen“ stark verliert, „Persönliche Selbständigkeit" und „Sicheres Selbstbewußtsein“ aber gewinnen, zeigt, daß nicht nur Werte auf höherem Abstraktionsgrad an die Stelle weniger abstrakter Werte treten, sondern zugleich auch persönliche an die Stelle kollektiver Werte treten: Zunehmen- 
de Selbständigkeit heißt auch zunehmende Durchsetzungsfreude, um nicht zu sagen: zunehmende Rücksichtslosigkeit.

Der gemessene Wandel in den schulischen Zielwerten legt nun Hypothesen über den Bedeutungswandel nahe. Wenn die kognitiven Zielwerte auf Kosten der evaluativen Zielwerte gewinnen, so könnten erstens die kognitiven Zielwerte auch in ihrer Bedeutungsstruktur näher aneinandergerückt sein. Wenn „Persönliche Selbständigkeit“ und „Sicheres Selbstbewußtsein“" gleichzeitig mit den kognitiven Zielwerten gewonnen haben, so könnten sie zweitens auch in der Bedeutungsstruktur näher an die kognitiven Zielwerte herangerückt sein. Wenn „Achtung vor den Mitmenschen“ zugunsten von "Persönlicher Selbständigkeit" und "Sicherem Selbstbewußtsein" an Boden verliert, so könnte sie drittens auch in der Bedeutungsstruktur sich von den Autonomie-Werten fort zu den Konventions-Werten hin bewegt haben; die Bedeutungskomponente autonomer Rücksichtnahme könnte hinter die Bedeutungskomponente angepaßter Konventionalität zurückgetreten sein.

Die Hypothesen über die Bedeutungsstruktur auf der einen Seite, den Bedeutungswandel auf der anderen Seite müssen nun unabhängig vom gemessenen Wandel empirisch überprüft werden. Dafür sind drei statistische Instrumente erforderlich: erstens ein Assoziationsmaß, das für jeden Zeitpunkt die Beziehungen zwischen zwei Werten unabhängig von der Randverteilung jedes Wertes erfaßt; zweitens ein Verfahren, das für jeden Zeitpunkt die Werte in allen möglichen bivariaten Beziehungen simultan darstellt; und drittens ein Verfahren, das die simultanen Darstellungen der Werte für jeden der drei Zeitpunkte miteinander vergleicht. Als Assoziationsmaß für die Beziehung zwischen zwei Werten verwende ich den Interaktionseffekt aus einem loglinearen Modell nach Goodman (1978), in dem die Zellenhäufigkeiten der Kreuztabelle als ein Effekt der Randverteilung der einen und der anderen Variablen sowie der Interaktion beider Variablen dargestellt werden. $\mathrm{Da}$ in einem solchen Modell die Effekte linearadditiv, d. h. voneinander unabhängig sind, stellt der Interaktionseffekt die Beziehung unabhängig von den Randverteilungen dar. Diese Interaktionseffekte wurden im ersten Schritt für jeden Zeitpunkt für alle $9 \times 8 / 2=36$ Vierfeldertafeln errechnet, in denen die Wahl/Nichtwahl eines Wertes mit der Wahl/Nichtwahl eines anderen Wertes kreuztabelliert war. Im zweiten Schritt wurden die Interaktionseffekte mit MINISSA (Roskam 1975) für jeden Zeitpunkt multidimensional skaliert. Im dritten Schritt schließlich wurden die Ergebnisse der multidimensionalen Skalierung, die grafische Darstellung der Beziehungen der Werte für jeden Zeitpunkt, mit PINDIS (Borg/Lingoes 1977) in einer Prokrustes-Rotation miteinander verglichen. $^{3}$

Der dritte Schritt ist das Ziel der Analyse, die im folgenden dargestellt wird. In ihm werden die beiden Arten von Hypothesen, über die Struktur und über den Wandel der Bedeutungen, überprüft. Erstens wird eine durchschnittliche Skalierung für alle Zeitpunkte erstellt und die Abweichung zwischen durchschnittlicher und einzelner Skalierung gemessen; damit wird die Struktur der Werte überprüft. Zweitens wird die Entwicklung jedes Wertes über die drei Zeitpunkte in einem Raum nachgezeichnet: Jeder Wert hinterläßt gleichsam eine Spur, die die Entwicklung zwischen 1958 und 1979 darstellt; damit wird der Wandel der Struktur überprüft. Kurzum: es werden die Struktur und der Strukturwandel der Werte erfaßt. Der Strukturwandel aber ist ein Indiz für den Bedeutungswandel der Werte.

\section{Bedeutungsstruktur und Bedeutungswandel in der gesamten Bevölkerung}

Die schulischen Zielwerte ließen sich in einer Kreuzung der Dichotomien evaluativ/kognitiv und abstrakt/konkret ordnen. Findet sich diese theoretische Ordnung in den empirischen Beziehungen zwischen den Werten zu jedem Zeitpunkt wieder? Um diese Frage zu beantworten, wurden die 36 Assoziationsmaße (Goodmans Lambdas für die Interaktion) zwischen den 9 Werten mit MINISSA multidimensional skaliert; für die Skalierung wurde schwache Monotonizität gefordert, d. h. es wurde zugelassen, daß3 gleiche Dissimilaritäten (Ausgangsdaten) durch benachbarte Distanzen (Ergebnis-Werte) und daß benachbarte Dissimilaritäten durch gleiche Distanzen wiedergegeben

\footnotetext{
${ }^{3}$ MINISSA und PINDIS wurden mit den Programmen aus der MDS(X)-Serie von Coxon/Jones/Tagg (1981), die log-linearen Modelle mit dem Programm ECTA von Goodman gerechnet. Einführende Darstellungen für MINISSA und PINDIS finden sich in Coxon (1982: 43-92 bzw. 202-221) und in Borg (1981: 51-92 bzw. 369-521). Für viele Hilfen und Hinweise bei der Anwendung von PINDIS danke ich Dr. Walter Bien (Wuppertal), Dr. Rolf Langeheine (Kiel) und Dipl.Psych. Detlef Leutner (Aachen).
} 
wurden. Die Zahl der Dimensionen ist durch die apriori-Klassifikation auf 2 festgelegt. ${ }^{4}$ Ist nun auch empirisch die Struktur der schulischen Zielwerte zweidimensional oder läßt sie sich - bei der geringen Zahl von nur 9 Vorgaben - auf eine Dimension zurückführen?

Diese Frage kann beantwortet werden, wenn man Skalierungen in unterschiedlicher Zahl von Dimensionen nach der Anpassung an die Ausgangsdaten miteinander vergleicht; ein $\mathrm{Ma} ß$ für die Güte der Anpassung ist der Streß, der für 3,2 und 1 Dimensionen und für alle drei Zeitpunkte in Tabelle 1 wiedergegeben ist; je kleiner der Wert, desto besser ist die Lösung den Daten angepaßt. Die Streß-Werte für 3 Dimensionen sind aufgrund der relativ geringen Zahl der Vorgaben extrem niedrig. Die Streß-Werte für 2 Dimensionen sind nach den Faustregeln für Streß-1 (Rabinowitz 1975) gerade noch als "gut“ zu bezeichnen; sie betragen im Durchschnitt etwa .10 und steigen gegenüber den dreidimensionalen Lösungen nicht erheblich an. Die Streß-Werte für eine Dimension dagegen sind nach den Faustregeln "schlecht" und steigen gegenüber den zweidimensionalen Lösungen erheblich an. Für jeden Zeitpunkt ist also die zweidimensionale Lösung angemessen, und in keinem Falle lassen sich die schulischen Zielwerte auf nur einer Dimension anordnen. Auch empirisch also müssen die schulischen Zielwerte in zwei Dimensionen dargestellt werden. Entspricht aber diese Darstellung der theoretischen Ordnung in vier Gruppen von Werten, und ist diese Ordnung über die drei Zeitpunkte konstant oder wandelt sie sich?

Tabelle 1 Streß-1-Werte mit Kruskals D-Hat* für 3 bis 1 Dimensionen zu verschiedenen Zeitpunkten

\begin{tabular}{c|c|c|c}
\hline \multirow{2}{*}{ Dimensionen } & \multicolumn{3}{|c}{ Zeitpunkte } \\
& 1958 & 1973 & 1979 \\
\hline 3 & .048 & .028 & .026 \\
2 & .111 & .074 & .104 \\
1 & .324 & .277 & .374 \\
\hline
\end{tabular}

* Schwache Monotonizität in den Distanzen und mögliche Auflösung verbundener Ausgangsdaten.

${ }^{4}$ Auch die üblichen Faustregeln - das Verhältnis von Datenpunkten zu Dimensionen darf 4 nicht überschreiten (Rabinowitz 1975); die Zahl der Dissimilaritäten soll mindestens doppelt so groß sein wie die Zahl aller Koordinatenwerte in der Lösung (Coxon 1982: 59,88) ließen nicht mehr als zwei Dimensionen zu.
Diese Frage kann beantwortet werden, wenn man die Ergebnisse der multidimensionalen Skalierungen, die zweidimensionalen Konfigurationen für jeden der drei Zeitpunkte in einer durchschnittlichen Konfiguration zusammenfaßt und die Anpassung der einzelnen Konfigurationen an die durchschnittliche Konfiguration überprüft. Ist die Anpassung gut, so kann man die durchschnittlichen Konfigurationen als über die Zeit konstantes Muster der Werte interpretieren. Gleichzeitig kann man die einzelnen Konfigurationen in die durchschnittliche Konfiguration eintragen und über drei Zeitpunkte gleichsam die Spur der Wertentwicklung nachzeichnen. Dies geschieht durch das Programm PINDIS, das die Konfigurationen für die drei Zeitpunkte als Eingabe übernimmt und sie so zu einer durchschnittlichen Konfiguration zusammenfaßt, daß die Abweichungen der Koordinatenwerte zwischen den Ausgangskonfigurationen und der Durchschnittskonfiguration nach dem Kriterium der kleinsten Quadrate minimiert werden. Dabei werden die Ausgangskonfigurationen lediglich „erlaubten" Transformationen unterworfen: der Rotation bzw. Reflexion, der Maßstabsveränderung und der Verlagerung des Ursprungs. Diese Transformationen heißen ,erlaubt", weil sie die wesentlichen Informationen der Ausgangskonfigurationen, die Größenrelationen zwischen den Distanzen, nicht verändern. Der minimierte Wert der quadrierten Abweichungen erfaßt die Güte der Anpassung jeder einzeinen Konfiguration an die Durchschnittskonfiguration. Die Korrelation der Koordinatenwerte zwischen jeder einzelnen und der durchschnittlichen Konfiguration - bzw. das als Kommunalität interpretierbare Quadrat dieser Korrelation - beschreibt, wie gut jede einzelne Konfiguration mit der Durchschnittskonfiguration übereinstimmt.

Die Kommunalitäten zwischen den zweidimensionalen Konfigurationen für 1958, 1973 und 1979 und der Durchschnittskonfiguration sind nun mit 91\%, 94\% und $93 \%$ sehr hoch; die zweidimensionale Konfiguration der drei Zeitpunkte überdeckt sich also fast vollständig mit der Durchschnittskonfiguration. Mit anderen Worten: die Struktur der schulischen Zielwerte ist über die Zeit konstant. ${ }^{5}$

${ }^{5}$ Dieser Eindruck ist offensichtlich von der bloßen Inspektion der Ergebnisse her. Aber auch eine an Simulationsstudien gewonnene Signifikanzprüfung zeigt, daß die Höhe der quadrierten Korrelation weit überzufällig ist - in einer entsprechenden, aus Zufallsdaten produzierten Konfiguration wäre nur in $5 \%$ aller Fälle ein $r^{2}$ über $.696 \mathrm{zu}$ erwarten (Langeheine 1980). 
Gemessen an der Struktur der Beziehungen zwischen den schulischen Zielwerten hat kein Bedeutungswandel zwischen 1958 und 1979 stattgefunden.

Die Durchschnittskonfiguration kann also als konstantes Bedeutungsmuster für 1958, 1973 und 1979 interpretiert werden. Sie ist in Abb. 1 wiedergegeben - mitsamt der Spur, die durch die Abfolge der Einzelkonfigurationen gebildet wird. Die Durchschnittskonfiguration bestätigt - sehr weitgehend, jedoch mit Einschränkungen - die theoretische Ordnung der Werte, die in Abbildung 1 durch die Zusammenfassung der Punkte mit einer durchge- zogenen Linie dargestellt ist. Das wird auf zwei Weisen sichtbar. Erstens erlaubt die Durchschnittskonfiguration - wie im übrigen auch jede Einzelkonfiguration - eine Zusammenfassung benachbarter Punkte, die genau der theoretischen Ordnung entspricht. Die kognitiv-konkreten Werte oder Fertigkeiten „Kenntnisse für den Beruf“ und „Vielseitiges Wissen“ sind auf der einen Seite benachbart dem kognitiv-abstrakten Wert oder der Kompetenz „Eigene Urteilsfähigkeit“, auf der anderen Seite den evaluativ-konkreten oder Konventions-Werten „Ordnung und Disziplin“ und „Gute Umgangsformen“. Kognitiv-abstrakte und evalua-

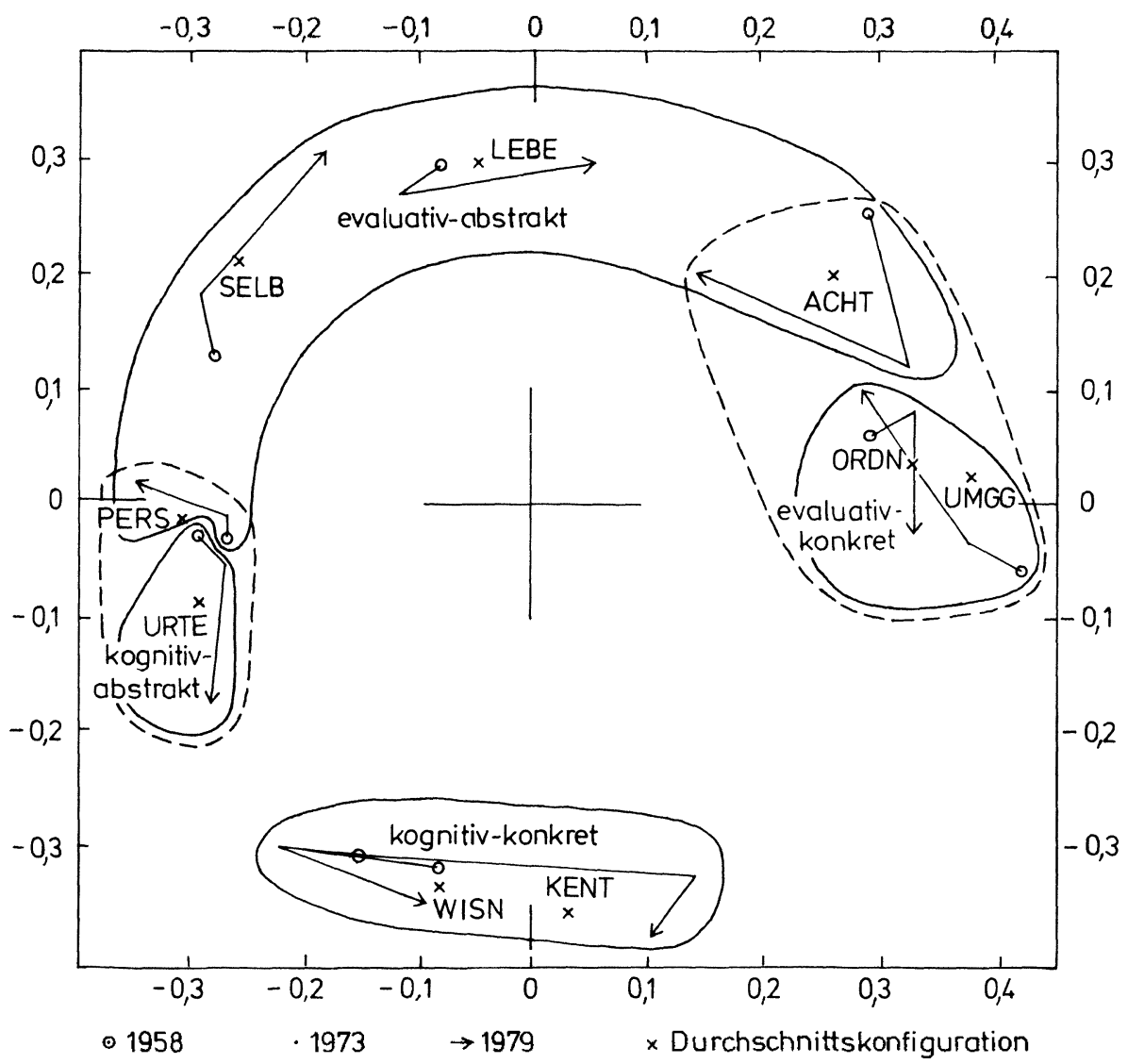

Abbildung 1 Die Bedeutungsstruktur schulischer Zielwerte 1958-1973-1979 in den Gesamtstichproben: Maximale Annäherung der Konfigurationen an die durchschnittliche Konfiguration mit Hilfe erlaubter Transformationen (PINDIS, Stufe PO)

KENT: Kenntnisse für den Beruf WISN: Vielseitiges Wissen URTE: Eigene Urteilsfähigkeit ORDN: Ordnung und Disziplin UMGG: Gute Umgangsformen
ACHT: Achtung vor den Mitmenschen

PERS: Persönliche Selbständigkeit

SELB: Sicheres Selbstbewußtsein

LEBE: Lebensfreude 
tiv-konkrete Werte umrahmen aber ihrerseits wiederum die evaluativ-abstrakten oder AutonomieWerte „Persönliche Selbständigkeit“, „Sicheres Selbstbewußtsein“, „Lebensfreude“ und „Achtung vor den Mitmenschen". Zweitens bildet die Verbindungslinie zwischen den Punkten der Durchschnittskonfiguration einen Circumplex (Lingoes/ Borg 1977), d. h. ein konvexes Vieleck ohne Einbrüche nach innen ${ }^{6}$, in dem sich die Werte von kognitiv-konkreten über kognitiv-abstrakte, evaluativ-abstrakte bis zu evaluativ-konkreten aneinanderreihen. Allerdings - und das sind die Einschränkungen - zieht die theoretische Ordnung der Werte die Grenzen nicht dort, wo empirisch die größten Klüfte liegen: nahe zusammenliegende Werte, wie „Persönliche Selbständigkeit" und „Eigene Urteilsfähigkeit", werden auseinandergerissen, voneinander entfernt liegende Werte, wie "Lebensfreude“ und „Achtung vor den Mitmenschen", zusammengefaßt. Die theoretische Ordnung wird zwar nicht widerlegt, aber sie trifft die empirische Durchschnittskonfiguration nicht optimal. Zwei Revisionen der theoretischen Ordnung durch die Durchschnittskonfiguration liegen nahe, die in Abbildung 1 durch gestrichelte Linien dargestellt sind.

Als erstes sollten „Persönliche Selbständigkeit“ und "Eigene Urteilsfähigkeit" zusammengefaßt werden. Beide Werte liegen auf einem hohen $\mathrm{Ab}$ straktionsniveau, beide beziehen sich - durch die Betonung von „persönlich“" oder von ,eigen“ - auf eine gewisse Innen-Lenkung. Auf der einen Seite kann man auch „Eigene Urteilsfähigkeit“ zu den evaluativen, spezifisch zu den Autonomie-Werten rechnen. Dafür spricht, daß mit „Urteil“ ein moralisches ebensogut wie ein kognitives gemeint sein kann (Meulemann 1982: Fn 5) Auf der anderen Seite kann man auch „Persönliche Selbständigkeit" zu den kognitiven Werten, spezifisch den Kompetenzen, rechnen. Dafür spricht, daß Selbständigkeit ebensogut als Fähigkeit wie als Charakterzug verstanden werden kann. Als zweites sollte „Achtung vor den Mitmenschen“ nicht als evaluativ-abstrakter, sondern als evaluativ-konkreter Wert verstanden und mit „Ordnung und Disziplin“ und „Gute Umgangsformen“ zusammengefaßt

${ }^{6}$ Die Konvexitätsbedingung verbietet Einbrüche des Circumplexes nach innen; es müssen von jedem Punkt innerhalb des Vielecks gerade Linien zu jedem Punkt gezogen werden können, ohne daß Verbindungslinien zwischen den Punkten zerschnitten werden (Lingoes/ Borg 1977: 138). Diese Bedingung ist nur an einer Stelle, für ORDN, und in schwachem Ausmaß verletzt. werden. „Achtung vor den Mitmenschen" wird offenbar nicht als eine autonome, sondern als eine konventionelle Orientierung an Kollektiven gesehen; anders gesagt: diese Unterscheidung ist relativ untergeordnet - relativ deshalb, weil „Achtung vor den Mitmenschen“ zwar recht nahe bei „Ordnung und Disziplin“ und „Gute Umgangsformen“ liegt, ihre Distanz zu den Autonomie-Werten aber geringer ist als die Distanz von „Ordnung und Disziplin“ und „Gute Umgangsformen“ zu den Autonomie-Werten. Beide Revisionen lassen die kognitiv-konkrete Gruppe von Werten unberührt. Die evaluativ-konkreten Werte werden durch „Achtung vor den Mitmenschen“ ergänzt. Alle übrigen Werte lassen sich entweder als Autonomie-Werte zusammenfassen oder in eine Gruppe kognitiv-abstrakter - „Eigene Urteilsfähigkeit" und „Persönliche Selbständigkeit" - und eine Gruppe evaluativ-abstrakter Werte - „Sicheres Selbstbewußtsein" und "Lebensfreude" - aufteilen.

Wenn die Beziehungen zwischen den schulischen Zielwerten im wesentlichen gleich bleiben, so sind damit die Hypothesen über die Struktur der Bedeutungen bestätigt, die Hypothesen über den Bedeutungswandel aber widerlegt. Trotzdem kann man anhand der Spur der Wertentwicklung in Abbildung 1 prüfen, ob sich die Hypothesen über den Wandel nicht zumindest im Ansatz stützen lassen.

Die erste Hypothese, daß die kognitiven Zielwerte - „Kenntnisse für den Beruf“, „Vielseitiges Wissen“ und „Eigene Urteilsfähigkeit" - enger zusammenrücken, gilt insofern, als „Eigene Urteilsfähigkeit" sich etwas näher an die beiden konkretkognitiven Werte heranbewegt; die Trennung zwischen konkret- und abstrakt-kognitiven Werten verliert also ihre Bedeutung, und „Eigene Urteilsfähigkeit" tendiert vom moralischen in den kognitiven Bereich. Zur zweiten Hypothese, daß „Persönliche Selbständigkeit“ und „Sicheres Selbstbewußtsein" an die kognitiven Werte heranrücken, findet sich das Gegenteil. Beide Werte rücken stärker zu den übrigen evaluativen Werten hin, und die Distanz zwischen „Persönliche Selbständigkeit“ und „Eigene Urteilsfähigkeit wird von beiden Seiten her größer. Sieht man die Trends zu beiden Hypothesen zusammen, so ergibt sich eine Tendenz zur Abgrenzung evaluativer von kognitiven Werten. Die dritte Hypothese, daß „Achtung vor den Mitmenschen" sich von den AutonomieWerten zu den Konventions-Werten bewege, findet nur für die Periode 1958-1973 etwas, für den gesamten Zeitraum aber keine Unterstützung. 
Zwischen 1958 und 1979 geht die durchschnittliche Distanz von „Achtung vor den Mitmenschen“ zu den Konventions-Werten um .04 zwar geringfügig, die Distanz zu den Autonomie-Werten um .20 jedoch viel stärker zurück. Auch bei einer gleichsam mikroskopischen Betrachtung kann keine Rede davon sein, daß „Achtung vor den Mitmenschen" an Wertschätzung verloren hat, weil Bedeutungskomponenten der Konvention an die Stelle der Autonomie getreten wären. Der allgemeine Rückgang der Distanzen deutet hier weniger auf einen Bedeutungswandel eines einzelnen Wertes als auf eine Vereinheitlichung der Bedeutung aller evaluativen Werte. Wenn die Bewegungen der Werte sich überhaupt zusammenfassend beschreiben lassen, so grenzen sich also kognitive und evaluative Werte voneinander $a b$ und rücken intern zusammen. Alle diese Bewegungen aber zerstören die für alle Zeitpunkte gleiche Struktur nicht, die in Abbildung 1 mit der Durchschnittskonfiguration erfaßt ist: Das entscheidende Ergebnis ist und bleibt die Konstanz der Bedeutungen schulischer Zielwerte zwischen 1958 und 1979. Ich werde im folgenden die Durchschnittskonfigurationen als Maßstab für verschiedene andere Konfigurationen nehmen.

\section{Bedeutungsstruktur und Bedeutungswandel in disaggregierten Teilstichproben}

Die Struktur der schulischen Zielwerte bleibt zwischen 1958 und 1979 in einem erstaunlich hohen Grade konstant, die Bedeutung der Werte hat sich nicht gewandelt. Allerdings gilt das nur für die gesamte Bevölkerung. Nun könnte es durchaus sein, daß spezifische Bevölkerungsgruppen ihr Verständnis schulischer Zielwerte durchaus geändert haben und daß sich die Wandlungen in diesen Gruppen gegenseitig aufheben (Hyman 1972: 223-226). Die Konstanz in der Bevölkerung würde dann einen Wandel in Bevölkerungsgruppen verschleiern. Ist die Stabilität der Bedeutungen in der Gesamtbevölkerung nur scheinbar, weil sie einen gegenläufigen Bedeutungswandel in verschiedenen Bevölkerungsgruppen verwischt, oder findet sich die Stabilität der Bevölkerung auch in wichtigen Bevölkerungssegmenten wieder? Zur Beantwortung dieser Frage müssen die Analysen, die bisher für die gesamten Stichproben durchgeführt wurden, in Teilstichproben wiederholt werden. Die Gesamtstichprobe muß nach Merkmalen disaggregiert werden, die die Verteilungen und möglicher- weise auch die Struktur der Werte beeinflussen. Welche Merkmale sind das?

Als erstes beeinflußt die Ausbildung die Wahl der schulischen Zielwerte; sie wurde in allen Datensätzen an der Grenze Volksschulabschluß dichotomisiert (Meulemann 1982a: 242-244, 1982b): Die besser Ausgebildeten wählen der Tendenz nach die Werte häufiger, die im Zeitablauf ansteigen „Eigene Urteilsfähigkeit“ und „Persönliche Selbständigkeit" - und die Werte seltener, die im Zeitablauf fallen - „Ordnung und Disziplin“, „Gute Umgangsformen“ und „Achtung vor den Mitmenschen"; in einem Falle jedoch kehrt sich diese Regel um - „Kenntnisse für den Beruf“ wird, obwohl im Zeitverlauf zunehmend, von den besser Ausgebildeten seltener gewählt -, und für die übrigen Zielwerte sind die Bildungsunterschiede gering. Insgesamt gilt, daß die Unterschiede zwischen den beiden Bildungsgruppen zu allen Zeitpunkten weitgehend gleich sind und daß entsprechend die allgemeine Entwicklung sich in beiden Bildungsgruppen - lediglich auf unterschiedlichem Niveau - unverändert wiederfindet. Aus den Trends des Meinungswandels würde man also für beide Bildungsgruppen die gleichen, schon für die Gesamtgruppen untersuchten Hypothesen des Bedeutungswandels entwickeln. Zeigt sich auch in den beiden Bildungsgruppen eine konstante Struktur der Werte oder findet man in den beiden Gruppen eine unterschiedliche Entwicklung der Bedeutungen, obwohl die Entwicklung der Meinungen weitgehend gleich ist?

Die Bildungsgruppen sind vollständige Aufgliederungen der gesamten Stichproben. Wie diese enthalten sie also zwei Formen des sozialen Wandels in sich: Wandel, der sich mit dem Alterungsproze $\beta$ einer Stichprobe von Personen des gleichen Geburtszeitraumes ergibt, und Wandel, der sich aus dem Auftauchen junger Bevölkerungsgruppen und dem natürlichen Aussterben alter Bevölkerungsgruppen ergibt. Die erste Form ergibt sich aus Wandlungen in einer gegebenen Bevölkerungsgruppe, die zweite Form aus dem Austausch der Mitglieder in der Bevölkerung. Um die erste Form des Wandels zu isolieren, muß man konstante Geburtskohorten, d. h. mit der Zeit älter werdende Gruppen betrachten; aus natürlichen Gründen können die ältesten Gruppen der frühen und die jüngsten Gruppen der späten Stichproben aber nicht über den ganzen Zeitraum verfolgt werden. In einer solchen Analyse von Kohorten wird also für keinen Zeitpunkt mehr die gesamte Stichprobe betrachtet. $\mathrm{Da}$ in den hier analysierten drei Stich- 
proben das Ausbildungsniveau über die Zeit selbst noch in der konstanten Geburtskohorte steigt, müssen altersspezifische Ausbildungskohorten betrachtet werden (Meulemann 1982a: 237-242).

Das Alter beeinflußt die Wahl der schulischen Zielwerte nun in einer der Ausbildung ähnlichen Form. Zwar wählen hier, der Tendenz nach in jeder Stichprobe, die jüngeren Gruppen die Werte, die im Zeitverlauf zunehmen, die älteren Gruppen die Werte, die im Zeitverlauf abnehmen. Aber in den ausbildungsspezifischen Alterskohorten zeigt sich im wesentlichen wiederum die gleiche Tendenz wie in den Gesamtstichproben (Meulemann 1982: Tab. 8). Aus den Trends des Meinungswandels würde man also auch für die ausbildungsspezifischen Alterskohorten die gleichen, schon für die Gesamtgruppe untersuchten Hypothesen des Bedeutungswandels entwickeln. Zeigt sich auch in den ausbildungsspezifischen Alterskohorten eine konstante Struktur der Werte, oder findet man in ihnen unterschiedliche Entwicklungen der Bedeutungen, obwohl die Entwicklung der Meinungen weitgehend gleich ist?

Ich werde im folgenden die Struktur der Wertbeziehungen zunächst für Ausbildungsgruppen (Abschnitt 4.1), dann für ausbildungsspezifische Alterskohorten (Abschnitt 4.2) darstellen. Methodisch gehe ich dabei in der gleichen Weise vor wie in den Gesamtgruppen - bis auf einen wichtigen Unterschied. Zwar werden wiederum für jede Teilstichprobe zuerst die Assoziationen zwischen allen Wertpaaren errechnet, zweitens multidimensional skaliert und drittens miteinander verglichen. Der Vergleich der Wertkonfigurationen, der Ergebnisse der multidimensionalen Skalierungen, ist jetzt aber nicht mehr intern, sondern extern: er ruht nicht auf einer induktiven, sondern einer deduktiven Vorgehensweise. Während aus den Konfigurationen für die drei Gesamtstichproben eine Durchschnittskonfiguration gewonnen wurde, wird diese Durchschnittskonfiguration nun als Maßstab für den Vergleich der Konfigurationen der Ausbildungsgruppen bzw. der ausbildungsspezifischen Alterskohorten vorgegeben. Es wird also geprüft, wieweit diese Konfigurationen einer Konfiguration angepaßt sind, die nicht aus ihnen selbst, sondern aus anderen Analysen ermittelt worden ist. In PINDIS wird nicht eine Durchschnittskonfiguration aus den eingegebenen Konfigurationen errechnet, sondern eine externe Konfiguration als Hypothese mit den eingegebenen Konfigurationen verglichen.

\subsection{Ausbildungsgruppen}

In den Gesamtgruppen war die Struktur der schulischen Zielwerte zweidimensional; gilt dies auch für die Ausbildungsgruppen? Tabelle 2 zeigt, da $\beta$ in beiden Ausbildungsgruppen für jeden Zeitpunkt die Streß-Werte beim Übergang von 3 auf 2 Dimensionen nur geringfügig, beim Übergang von 2 auf 1 Dimension aber stark ansteigen? Auch in den Ausbildungsgruppen werden die Zielwerte also am besten in zwei Dimensionen dargestellt, aber sie können sich durchaus noch in der Konfiguration unterscheiden. Wieweit lassen sich nun die Konfigurationen in den Ausbildungsgruppen durch erlaubte Transformationen in die Durchschnittskonfiguration für die drei Gesamtstichproben, die Hypothesenkonfiguration, übersetzen? Darauf gibt die PINDIS-Analyse (Tab. 3) eine überraschende Antwort.

Die Konfigurationen für die weniger gut ausgebildeten Gruppen stimmen mit der Durchschnittskonfiguration zu jedem Zeitpunkt nahezu vollständig überein; die Konfigurationen für die besser ausgebildeten Gruppen stimmen dagegen mit der Durchschnittskonfiguration sehr viel schlechter überein: Vor allem 1958 mit .50, aber auch 1979 mit .73 ist die Übereinstimmung schwach, während sie 1973 mit .94 so stark wie in den weniger gut ausgebildeten Gruppen ist. ${ }^{8}$ In der Gruppe mit Hauptschulabschluß hat also die gleiche Entwicklung wie in der Gesamtgruppe stattgefunden: Konstanz der Wertbedeutung. In der Gruppe mit mehr als Hauptschulabschluß aber hat sich die Wertbedeutung gewandelt. Allein die Gruppe mit mehr als Hauptschulabschluß muß also im folgenden noch weiter untersucht werden. Wie läßt sich der Bedeutungswandel der schulischen Zielwerte zwischen 1958 und 1979 in der besser ausgebildeten Gruppe beschreiben? Dazu soll die Entwicklung

\footnotetext{
7 Um der Gefahr „lokaler Minima“ zu entgehen, wurden die zweidimensionalen MINISSA-Analysen mit der Durchschnittskonfiguration der Gesamtstichproben als Startkonfiguration wiederholt. Es ergaben sich die gleichen oder höhere, nicht aber niedrigere Streß-Werte. Die vorliegenden zweidimensionalen Lösungen sind also optimal.

${ }^{8}$ Die Variation der Kommunalitäten hängt nicht mit der Variation der Stichprobengröße zusammen. Jede ausbildungsspezifische Stichprobe wird nämlich mit der Durchschnittskonfiguration aus den Gesamtstichproben und nicht mit einer Durchschnittskonfiguration aus den Stichproben gleicher Ausbildung verglichen.
} 
Tabelle 2 Streß-1-Werte mit Kruskals D-Hat ${ }^{1}$ für 3 bis 1 Dimensionen in bildungsspezifischen Gruppen für jeden Zeitpunkt

\begin{tabular}{|c|c|c|c|c|c|c|}
\hline \multirow{3}{*}{ Dimensionen } & \multicolumn{6}{|c|}{ Ausbildung und Zeitpunkt } \\
\hline & \multicolumn{3}{|c|}{ Bis Volksschulabschluß } & \multicolumn{3}{|c|}{ Mehr als Volksschulabschluß } \\
\hline & 1958 & 1973 & 1979 & 1958 & 1973 & 1979 \\
\hline 3 & .071 & .028 & .029 & .032 & .041 & .035 \\
\hline 2 & .129 & .084 & .102 & .110 & .104 & .127 \\
\hline 1 & .318 & .249 & .319 & .296 & .276 & .345 \\
\hline
\end{tabular}

1 Siehe Tab. 1

Tabelle 3 Kommunalitäten $\left(r^{2}\right)$ der Konfigurationen für bildungsspezifische Gruppen zu jedem Zeitpunkt mit der Durchschnittskonfiguration

\begin{tabular}{c|c|c|c|c}
\hline \multirow{2}{*}{$\begin{array}{c}\text { Ausbildung, } \\
\text { Jahr }\end{array}$} & \multicolumn{3}{|c|}{ Hauptschulabschluß } & \multicolumn{3}{|c}{ Mehr als Hauptschulabschluß } \\
& $(\mathrm{n})$ & $\mathrm{r}^{2}$ & $(\mathrm{n})$ & $\mathrm{r}^{2}$ \\
\hline 1958 & $(1445)$ & .90 & $(329)$ & .50 \\
1973 & $(2803)$ & .92 & $(1111)$ & .94 \\
1979 & $(1206)$ & .91 & $(766)$ & .73 \\
\hline
\end{tabular}

der Werte wiederum grafisch über die drei Zeitpunkte verfolgt werden.

In Abb. 2 ist - exakt so wie in Abb. 1 - die Durchschnittskonfiguration für die Gesamtgruppen dargestellt. Anders als in Abb. 1 sind jetzt aber nicht mehr die drei Konfigurationen für die Gesamtgruppe, sondern für die Gruppe mit mehr als Hauptschulabschluß dargestellt, die den erlaubten Transformationen einer PINDIS-Analyse unterworfen sind. Schon auf den ersten Blick ist die Entwicklung in der besser ausgebildeten Gruppe nicht so einheitlich wie in den Gesamtstichproben. Aber es ist nur ein Wert, der seine Position deutlich verändert, während alle übrigen Werte sich fast so wenig bewegen wie in den Gesamtstichproben. ${ }^{9}$ „Kenntnisse für den Beruf“ liegt 1958 zwischen "Sicheres Selbstbewußtsein" und „Lebensfreude“, 1973 und 1979 aber zwischen „Vielseitiges Wissen“ und „Ordnung und Disziplin“; gemessen an der Durchschnittskonfiguration wandern „Kenntnisse für den Beruf“ quer über das ganze Feld, von den evaluativ-abstrakten zu den kognitiv-konkreten Werten - von einem Platz, wo man sie gar nicht erwarten würde, zu einem Platz, wo sie eigentlich hingehören. ${ }^{10}$ An einer Stelle also wird die Struktur der schulischen Zielwerte deutlich durchbrochen; an den übrigen Stellen wird die Struktur zwar nicht zerstört, aber es zeigt sich etwas mehr Bewegung als in den Gesamtgruppen.
Ich will kurz auf die Bewegungen innerhalb der Struktur eingehen, um dann ausführlich die Durchbrechung der Struktur durch „Kenntnisse für den Beruf“" $1958 \mathrm{zu}$ interpretieren.

9 Aus Abb. 2 wird weiterhin ersichtlich, warum die Konfiguration der besser ausgebildeten Gruppe 1979 sich mit einer Kommunalität von .73 nur relativ schlecht durch die Durchschnittskonfiguration der Gesamtgruppen wiedergeben läßt. Verbindet man für diese Gruppe die Punkte von „Kenntnisse für den Beruf“" bis „Umgang mit den Mitmenschen" in der Abfolge des Circumplexes der Durchschnittskonfiguration und vergleicht das entstandene Gebilde mit dem Circumplex der Durchschnittskonfiguration, so zeigen sich zwei Inversionen: zwischen „Persönliche Selbständigkeit“ und „Sicheres Selbstbewußtsein“ und zwischen „Ordnung und Disziplin" und „Umgang mit den Mitmenschen". Die Inversionen der Werte aber bewegen sich innerhalb der Gruppen der theoretischen Ordnung, so daß die Aufteilung der Werte nach abstrakt-konkret und evaluativ-konkret auch für die besser ausgebildete Gruppe 1979 gültig bleibt.

${ }^{10}$ Die Abweichung für KENT in der Gruppe mit mehr als Hauptschulabschluß 195 beruht nicht auf einer zufälligen, durch ein "lokales" Minimum bedingten MINISSA-Lösung. Wurde die Durchschnittskonfiguration der Gesamtgruppen als Startkonfiguration vorgegeben, so erhöhte sich der Streß-Wert von .111 auf .141 (siehe auch Fußnote 7). 


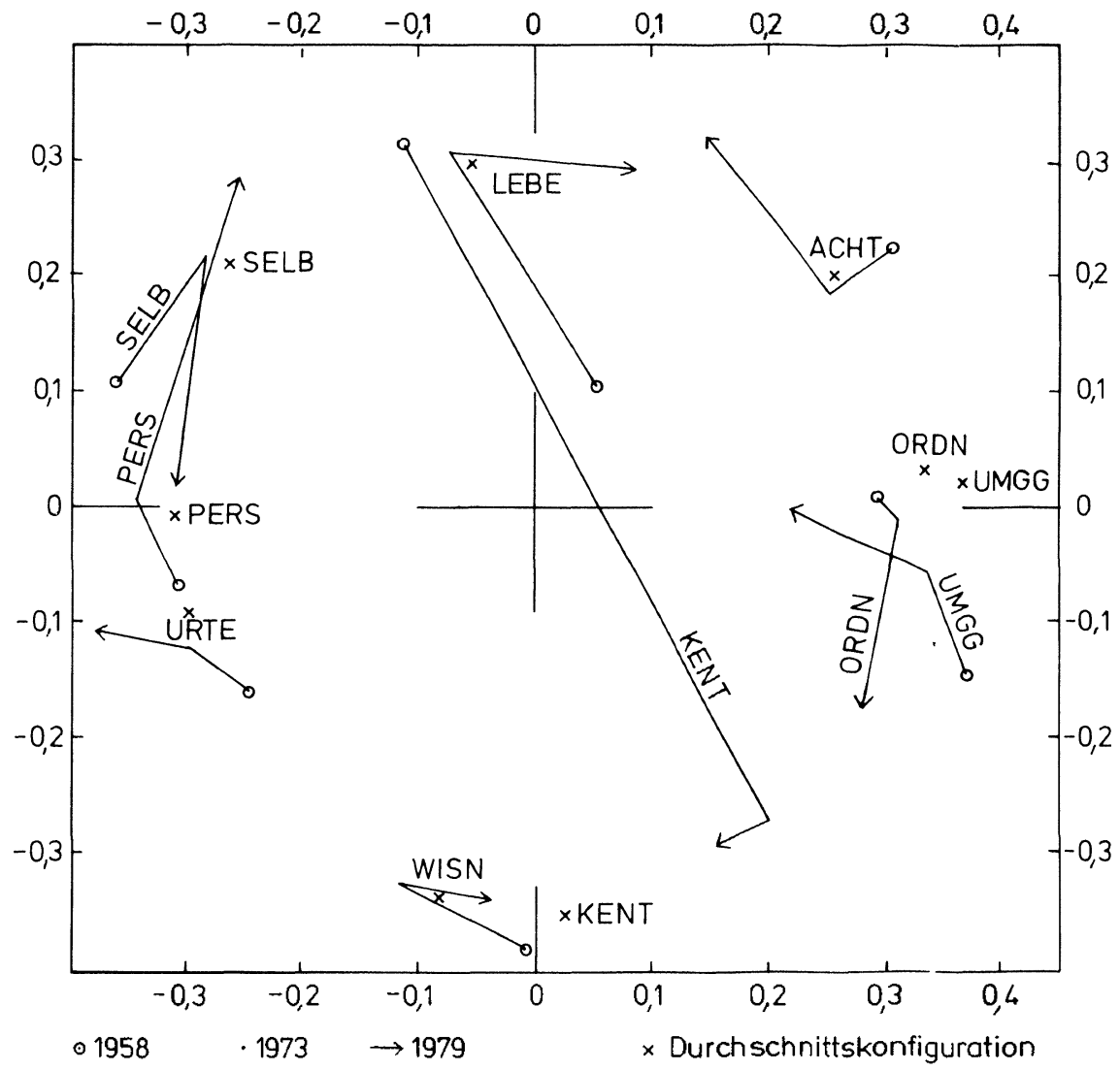

Abbildung 2 Die Bedeutungsstruktur schulischer Zielwerte 1958-1973-1979 in den Gruppen mit mehr als Hauptschulabschluß: Maximale Annäherung der Konfigurationen an die durchschnittliche Konfiguration der Gesamtstichproben mit Hilfe erlaubter Transformationen (PINDIS, Stufe PO)

KENT: Kenntnisse für den Beruf

WISN: Vielseitiges Wissen

URTE: Eigene Urteilsfähigkeit

ORDN: Ordnung und Disziplin

UMGG: Gute Umgangsformen
ACHT: Achtung vor den Mitmenschen

PERS: Persönliche Selbständigkeit

SELB: Sicheres Selbstbewußtsein

LEBE: Lebensfreude
Die Bewegungen der Werte lassen sich mit Distanzen zwischen Werten und Wertgruppen beschreiben, die in Tab. 4, geordnet nach den Hypothesen über den Bedeutungswandel, dargestellt sind. Die erste Hypothese wird bestätigt: die kognitiven Werte rücken, mit dem Anstieg ihrer Nennung, auch in ihrer Bedeutung zusammen. Während „,Kenntnisse für den Beruf“ und „Vielseitiges Wissen" 1958 die größte Distanz von allen (nicht nur den kognitiven) Paaren aufweisen, rücken sie 1973 ins Mittelfeld und 1979 an die zweitletzte Stelle der Distanzen. Die beiden kognitiv-konkreten Werte rücken also zusammen. Auf der anderen Seite aber rückt der kognitiv-abstrakte Wert „Eigene Urteils- fähigkeit“ von den beiden kognitiv-konkreten Werten ab. Die Distanz zwischen „Eigene Urteilsfähigkeit" und „Vielseitiges Wissen“ - dem „Eigene Urteilsfähigkeit“" näherliegenden kognitiv-konkreten Wert - ist 1979 viel größer als 1958 und 1973. Allerdings ist diese Differenzierung der kognitiven Werte weniger ausgeprägt und zeitlich später als die Vereinheitlichung der kognitiven Werte: zwischen 1958 und 1973 haben sich die kognitiv-konkreten Werte als Gruppe herausgebildet, um sich zwischen 1973 und 1979 von dem kognitiv-abstrakten Wert zu entfernen. Insgesamt aber haben sich die kognitiven Werte - wie der Durchschnitt der Distanzen zeigt - in ihrer Bedeu- 
tung einander angenähert; in der besser ausgebildeten zeigt sich also hier das gleiche Ergebnis wie in der Gesamtgruppe. Die zweite Hypothese wird deutlich widerlegt: „Persönliche Selbständigkeit“" und „Sicheres Selbstbewußtsein“ rücken nicht an die kognitiven Zielwerte heran, sondern von ihnen weg; auch hier zeigt sich in der besser ausgebildeten das gleiche Ergebnis wie in der Gesamtgruppe. Die dritte Hypothese wird widerlegt: „Achtung vor den Mitmenschen" rückt von den Konventions-Werten etwas ab und an die AutonomieWerte etwas heran. Auch in der besser ausgebildeten Gruppe kann also keine Rede davon sein, daß „Achtung vor den Mitmenschen“" an Wertschätzung verloren hat, weil Bedeutungskomponenten der Konvention an Stelle der Autonomie getreten wären. Auch in der besser ausgebildeten Gruppe hat sich weniger ein einzelner evaluativer Wert verlagert als die Bedeutung der evaluativen Werte insgesamt vereinheitlicht.

Die Bewegungen der Werte in der besser ausgebildeten Gruppe lassen sich also in der gleichen Weise zusammenfassen wie in der Gesamtgruppe: kognitive und evaluative Werte rücken voneinander $\mathrm{ab}$ und intern zusammen. Die Bewegungen sind jedoch etwas stärker als in der Gesamtgruppe und durchbrechen an einer Stelle die Bedeutungsstruktur: „Kenntnisse für den Beruf“ wechselt zwischen 1958 und 1973 von den evaluativ-abstrakten zu den kognitiv-konkreten Werten (Abb. 1) und verringert deutlich seine Distanz zu ,Vielseitiges Wissen“ (Tab. 4). Wie läßt sich diese Entwicklung interpretieren?

Die Personen mit mehr als Hauptschulabschluß sind zwar eine elitäre Gruppe, aber sie ist im Laufe der hier betrachteten Zeit immer weniger elitär geworden. Während 1958 nur 18\% der Stichprobe mindestens Mittlere Reife hatten, waren es 1973 fast $30 \%$ und 1979 fast $40 \%$ der Stichprobe; zwi- schen 1958 und 1973 tritt die Bildungsexpansion, die den relativen Wert von Bildung mindert. „Kenntnisse für den Beruf" waren nun in den höheren Institutionen des allgemeinbildenden Schulwesens ein eher peripheres Lernziel: Sie werden in der Lebenspraxis erworben, von der sich die Allgemeinbildung ja gerade abhebt. Dies gilt um so stärker, je mehr die Allgemeinbildung von ihrer pädagogischen Konzeption und von ihrer sozialen Rekrutierung her elitär ist. Mit der Bildungsexpansion aber haben die elitären Konzeptionen der Allgemeinbildung - z. B. die pädagogische Rechtfertigung des dreigliedrigen Aufbaus der Sekundarstufe - ihre Verbindlichkeit verloren, und die weiterführenden Zweige des Schulwesens haben ihre soziale Basis stark erweitert. Die elitäre Abschottung der Allgemeinbildung ist zurückgegangen, und eine instrumentelle Einstellung zur Schule als eines Mittels des beruflichen und sozialen Aufstiegs ist an ihre Stelle getreten.

Auf diesem Hintergrund wird der Bedeutungswandel der „Kenntnisse für den Beruf“ in der besser ausgebildeten Gruppe verständlich. Erst mit der Bildungsexpansion haben die Bildungsvorstellungen dieser Gruppe die der Allgemeinheit eingeholt. Im traditionellen Verständnis der gebildeten Gruppen waren „Kenntnisse für den Beruf“ weit entfernt von semantisch ähnlichen kognitiven Werten wie „Vielseitiges Wissen“ und „Eigene Urteilsfähigkeit" und eng mit den Werten der Lebenspraxis wie „Sicheres Selbstbewußtsein“, „Lebensfreu-

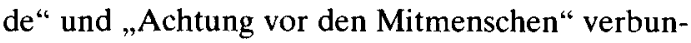
den. „Kenntnisse für den Beruf“ waren vom zweckfreien, „vielseitigen“ Wissen maximal distanziert. Während sich aber die Konturen der gebildeten Gruppen verwischen, verschwindet auch die Distanzierung zwischen beruflichen Kenntnissen und allgemeinem Wissen. Die Bildung wird zur Ausbildung, die Schule zum Instrument des Berufes. In den Gruppen mit Haupt-

Tabelle 4 Distanzen zwischen schulischen Zielwerten 1958-1973-1979 für Befragte mit mehr als Hauptschulabschluß, geordnet nach den Hypothesen über den Bedeutungswandel (siehe Abb. 2)

\begin{tabular}{c|c|c|c|c|c|c|c|c}
\hline \multirow{2}{*}{ Jahr } & \multicolumn{3}{|c|}{ 1. Hypothese: Kognitive Zielwerte } & \multicolumn{2}{c|}{ 2. Hypothese } & \multicolumn{4}{c}{ 3. Hypothese } \\
& $\begin{array}{c}\text { KENT } \\
\text { WISN }\end{array}$ & $\begin{array}{c}\text { KENT } \\
\text { URTE }\end{array}$ & $\begin{array}{c}\text { WISN } \\
\text { URTE }\end{array}$ & $\begin{array}{c}\text { Durch- } \\
\text { schnitt }\end{array}$ & $\begin{array}{c}\text { PERS + SELB } \\
\text { Kognitive Werte }\end{array}$ & $\begin{array}{c}\text { ACHT } \\
\text { Konvention }\end{array}$ & $\begin{array}{c}\text { ACHT } \\
\text { Autonomie }\end{array}$ & $\begin{array}{c}\text { Durchschnitt } \\
\text { evaluative } \\
\text { Werte }\end{array}$ \\
\hline 1958 & .71 & .50 & .33 & .51 & .37 & .30 & .55 & .45 \\
1973 & .33 & .52 & .27 & .37 & .48 & .23 & .50 & .43 \\
1979 & .13 & .57 & .48 & .39 & .50 & .42 & .34 & .42 \\
\hline
\end{tabular}

Die Werte zur 2. und 3. Hypothese sind Durchschnitte der jeweiligen Einzeldistanzen 
schulabschluß aber, die sich ja weitgehend mit den unteren sozialen Schichten überschneiden, wurde nie zwischen Zweck und Selbstzweck der Bildung getrennt. Vom Schuleintritt an hatte und hat man den Hauptschulabschluß und mit ihm den Beruf im Visier. Die Bildungsexpansion hat daran nichts geändert - lediglich, daß man sich weiterreichende berufliche Ziele steckt. Mit einem Wort: Die Bildungsexpansion zwingt die oberen sozialen Straten, sich in ihrer Bildungsvorstellung den unteren anzupassen, und nicht umgekehrt. ${ }^{11}$

Wenn nun die breitere soziale Rekrutierung höherer Ausbildungen den Bedeutungswandel bewirkt hat, könnte man vermuten, daß nur die neu hinzugekommenen Gruppen die neuen Bedeutungen tragen, während die Gruppen, die schon über Generationen höhere Ausbildung genießen, die alte Bedeutung hochgehalten haben. Diese Vermutung läßt sich prüfen, wenn man für die beiden Zeitpunkte nach der Bildungsexpansion, 1973 und 1979, aus der besser ausgebildeten Gruppe diejenigen herausgreift, deren Väter schon mindestens einen Realschulabschlu $\beta$ hatten, und mit dieser Gruppe wiederum die gleichen Analyseschritte durchführt. Die multidimensionale Skalierung der

${ }^{11}$ Man kann die Entwicklung in den besser ausgebildeten Gruppen als eine Desillusionierung verstehen. Man kann sie als eine Auflösung eines unangebrachten Dünkels begrüßen oder als die Erosion wichtiger Werte -.,Bildung" ist zur "Ausbildung" herabgekommen beklagen. Die Interpretation, die hier angedeutet wurde, schließt keine derartige Wertung ein. Weil hier ein altes Thema deutschen Erziehungsdenkens und deutscher Schulpraxis angesprochen wird, ist es vielleicht wichtig, diese Selbstverständlichkeit zu betonen. Es geht nicht um Ideologiekritik oder um Kulturpessimismus, sondern um die Analyse realer Entwicklungen. Weiterhin ist der Hinweis wichtig, daß die Analyse nur auf Vorstellungen, nicht auf tatsächliche Funktionen gerichtet ist. Sicher hat die höhere Bildung auch früher Zwecken gedient, und sicher hätten Personen mit besserer Schulbildung, darauf angesprochen, die Zweckmäßigkeit von Bildung durchaus anerkannt und zugegeben. Aber sie hielten eine andere Vorstellung von Bildung aufrecht. Und sie konnten eine andere Vorstellung aufrechterhalten, weil die institutionellen Bedingungen das Bildungswesen vor der Bildungsexpansion nicht dazu zwangen, sich das Selbstverständliche bewußt zu machen. Wenn mit Bildungsabschlüssen gleichsam natürlich bestimmte Berufspositionen verbunden sind, dann ist der Nutzen von Bildung selbstverständlich, und ihr Eigenwert tritt in den Vordergrund. Wenn aber die höheren Abschlüsse ansteigen, dann muß um Berufspositionen konkurriert werden; der Nutzen von Bildung und die „soziale Planierung" werden zum Thema.
Assoziationen (Goodmans-Lambda) der Werte in diesen besser ausgebildeten und konsistenten Gruppen zeigte nur wenig höhere Streß-Werte als in den besser ausgebildeten Gruppen überhaupt (siehe Tab. 2). Stimmt die Konfiguration der besser ausgebildeten konsistenten Gruppen 1973 und 1979 nun eher mit der besser ausgebildeten Gruppe 1958 oder mit der Durchschnittskonfiguration überein?

PINDIS-Analysen der beiden konsistenten Gruppen, in denen entweder die besser ausgebildete Gruppe 1958 oder die Durchschnittskonfiguration als Hypothese vorgegeben wurde (Tab. 5), zeigen weit geringere Kommunalitäten für die besser ausgebildete Gruppe 1958. Die Kommunalitäten für die besser ausgebildeten konsistenten Gruppen in Tab. 5 sind nahezu identisch mit den Kommunalitäten für die besser ausgebildeten Gruppen insgesamt in Tab. 3. Auch in der konsistenten hat also dieselbe Entwicklung stattgefunden wie in der gesamten besser ausgebildeten Gruppe. Wie eine genauere Inspektion zeigt, sind 1973 die Konfigurationen fast identisch, und 1979 teilt die Konfiguration der konsistenten die Unregelmäßigkeiten der Konfiguration der gesamten besser ausgebildeten Gruppe. Hätte man also in Abb. 2 statt der Punkte für die Gesamtgruppe die Punkte für die konsistente Gruppe eingetragen, so hätten sich 1973 keine und 1979 nur geringfügige Änderungen ergeben. Vor allem aber wäre die entscheidende Positionsveränderung der „Kenntnisse für den Beruf" in der gleichen Weise aufgetreten. ${ }^{12}$

Tabelle 5 Kommunalitäten $\left(r^{2}\right)$ der zweidimensionalen Konfiguration der konsistenten Gruppen mit mehr als Hauptschulabschluß 1973 und 1979 mit (a) der Konfiguration der Gruppe mit mehr als Hauptschulabschluß 1958 und (b) der Durchschnittskonfiguration

\begin{tabular}{c|c|c|c|c}
\hline Jahr & $(n)$ & $(\%)^{*}$ & $(a)$ & $(b)$ \\
\hline 1973 & $(539)$ & $(48,5)$ & .46 & .94 \\
1976 & $(322)$ & $(42,0)$ & .25 & .69 \\
\hline
\end{tabular}

* Basis ist $n$ mit mehr als Hauptschulabschluß in Tab. 3

${ }^{12}$ Als möglicher Einwand gegen diese Analyse der konsistenten Ausbildungsgruppen bleibt bestehen, daß eine Dichotomisierung der Bildung beim Realschulabschluß zu niedrig angesetzt ist, um traditionelle Bildungsgruppen herauszugreifen. Aber eine Bildung konsistenter Abiturienten- oder Akademikergruppen über zwei Generationen hätte 1973 und 1979 die Stichproben zu sehr verkleinert. 


\subsection{Ausbildungsspezifische Alterskohorten}

Anders als die Ausbildungsgruppen umfassen die ausbildungsspezifischen Alterskohorten nicht mehr die gesamten Stichproben, sondern nur noch die Personen, die den ganzen betrachteten Zeitraum durchlebt haben. ${ }^{13}$ Durch die Kohorten wird also der Erfahrungshintergrund kontrolliert, der mit dem Aufwachsen in einer bestimmten Periode und dem Durchgang durch das Bildungswesen einer bestimmten Periode bedingt ist. Durch die zusätzliche Kontrolle der Ausbildung werden die spezifischen Chancen der Verarbeitung solcher Erfahrungen kontrolliert, die mit einem höheren oder niederen Bildungsstand verbunden sind. $\mathrm{Da}$ im folgenden also sehr viel spezifischere Erfahrungslagen und gleichzeitig sehr viel kleinere Stichproben als bisher betrachtet werden, wird die Ausgangsfrage nach Konstanz oder Wandel der Bedeutung der Werte einem sehr viel härteren Test unterworfen.

Beginnt man wieder mit der Dimensionalität der schulischen Zielwerte (Tab. 6), so ist auch in allen ausbildungsspezifischen Alterskohorten die Struk- tur zweidimensional: Die Streß-Werte verschlechtern sich nur geringfügig beim Übergang von 3 auf 2 , aber deutlich beim Übergang von 2 auf 1 Dimensionen. Allerdings sind im Vergleich mit den Gesamtgruppen (Tab. 1) und mit den Ausbildungsgruppen (Tab. 2) die Streß-Werte für die ausbildungsspezifischen Alterskohorten doch erkennbar höher: sie liegen nicht mehr bei .10, son-

${ }^{13}$ Anders als in der Analyse der Gesamtgruppen und der Ausbildungsgruppen wird also im folgenden auch die Komposition der Bevölkerung kontrolliert, zumindest nach den sehr wichtigen Merkmalen Alter und Ausbildung. Die Meinung in der gesamten Gruppe kann sich allein dadurch wandeln, da $B$ sich die Zusammensetzung der Gesamtgruppe nach wichtigen Einflußvariablen der Meinung ändert (Davis 1975); was für die Verteilung einer abhängigen Variablen gilt, gilt natürlich auch für die Beziehungen zwischen mehreren abhängigen Variablen, d. h. den Bedeutungswandel. Durch die Kontrolle möglicher Kompositionseffekte gewinnen die folgenden Analysen ihren besonderen Stellenwert gegenüber den vorausgehenden.

Tabelle 6 Streß-1-Werte mit Kruskals D-Hat ${ }^{1}$ für 3 bis 1 Dimensionen in ausbildungsspezifischen Alterskohorten für jeden Zeitpunkt

\begin{tabular}{|c|c|c|c|c|c|c|c|c|}
\hline \multirow{3}{*}{\multicolumn{2}{|c|}{ Kohorte }} & \multirow[b]{3}{*}{ Dim } & \multicolumn{6}{|c|}{ Ausbildung und Zeitpunkt } \\
\hline & & & \multicolumn{3}{|c|}{ bis Volksschulabschluß } & \multicolumn{3}{|c|}{ mehr als Volksschulabschluß } \\
\hline & & & 1958 & 1973 & 1979 & 1958 & 1973 & 1979 \\
\hline \multirow[t]{3}{*}{ A } & 1958: $16-20$ & 3 & .074 & .061 & .048 & .094 & .058 & .055 \\
\hline & 1973: $25-34$ & 2 & $.153^{\star}$ & $.158^{\star}$ & .134 & .140 & .134 & .134 \\
\hline & 1979: $31-40$ & 1 & .389 & .326 & .320 & .323 & .305 & .298 \\
\hline \multirow[t]{3}{*}{ B } & 1958: $21-34$ & 3 & .096 & .050 & .040 & .052 & .059 & .075 \\
\hline & 1973: $35-49$ & 2 & $.185^{\star \star}$ & .137 & .105 & $.164^{\star}$ & .118 & $.151^{\star}$ \\
\hline & 1979: $41-55$ & 1 & .389 & .334 & .315 & .298 & .230 & .357 \\
\hline \multirow[t]{3}{*}{ C } & 1958: $35-49$ & 3 & .073 & .029 & .056 & .087 & .030 & .040 \\
\hline & 1973: $50-64$ & 2 & $.156^{\star}$ & .082 & $.151^{*}$ & $.185^{\star \star}$ & .113 & .149 \\
\hline & 1979: $56-70$ & 1 & .339 & .307 & .359 & .367 & .298 & .342 \\
\hline \multirow[t]{3}{*}{ D } & 1958: $50-64$ & 3 & .076 & .048 & .060 & .051 & .089 & .079 \\
\hline & 1973: $65+$ & 2 & $.179^{\star * *}$ & .106 & .131 & $.155^{\star}$ & $.186^{\star \star *}$ & $.170^{* *}$ \\
\hline & 1979: $71+$ & 1 & .336 & .329 & .293 & .367 & .403 & .399 \\
\hline
\end{tabular}

1 Siehe Tab. 1

für 2 Dimensionen: * Streß $>.150 ;{ }^{* *}$ Streß $>.170$ 
dern bei $.15 .{ }^{14}$ Die besonders hohen Streß-Werte konzentrieren sich weder in bestimmten Ausbildungsgruppen noch in bestimmten Kohorten, sondern in bestimmten Zeitpunkten: 1958 und $1979 .{ }^{15}$ Wieweit lassen sich nun die Konfigurationen in den ausbildungsspezifischen Alterskohorten durch erlaubte Transformationen in die Durchschnittskonfiguration für die Gesamtstichproben übersetzen? Darauf gibt die PINDIS-Analyse (Tab. 7) eine Antwort.

Die Kommunalität für die ausbildungsspezifischen Alterskohorten liegt im Durchschnitt erheblich unter der für die Ausbildungsgruppen. Welche Kohorten aber sind im einzelnen für die geringe durchschnittliche Kommunalität verantwortlich? Als erstes fällt auf, daß die Kohorten mit Hauptschulabschluß durchweg besser mit der Durchschnittskonfiguration übereinstimmen als die Kohorten mit mehr als Hauptschulabschluß. Das bestätigt die Ergebnisse des letzten Abschnitts noch

${ }^{14}$ Auch hier wurden, wie bei den Ausbildungsgruppen, die zweidimensionalen MINISSA-Analysen mit der Durchschnittskonfiguration der Gesamtstichproben als Startkonfiguration wiederholt, um der Gefahr ,lokaler Minima" zu entgehen. Auch hier zeigten sich in allen Fällen gleich große oder größere Streß-Werte bis auf eine Ausnahme: In der Kohorte $\mathrm{C}$ mit mehr als Volksschulabschluß hatte die Lösung mit einer zufälligen Startkonfiguration einen Streß von .209, die Lösung mit der Durchschnittskonfiguration als Startkonfiguration einen Streß von .185. Ich habe in diesem Falle die Lösung mit dem niedrigeren Streß-Wert in die weiteren PINDIS-Analysen eingegeben.

${ }^{15}$ Möglicherweise hängt das damit zusammen, daß die Stichprobe 1973 doppelt so groB ist wie die Stichprobe 1958 und 1979; auch schon bei der Analyse der Gesamtgruppen (Tab. 1) und der Ausbildungsgruppen (Tab. 2) wies die Stichprobe 1973 die niedrigsten StreB-Werte auf. Allerdings hängen die Streß-Werte nicht unmittelbar von der Stichprobengröße ab: eine gegebene Matrix von Assoziationskoeffizienten führt zu einem bestimmten Streß-Wert, einerlei wie groß die Stichprobe war. Mit größeren Stichproben wird es allerdings weniger wahrscheinlich, daB einzelne Assoziationskoeffizienten rein zufällig von ihrem wahren Wert abweichen. Auf diesem indirekten Wege könnte die Stichprobengröße tatsächlich Einfluß auf die Konfigurationen haben - vor allem bei einer starken Disaggregierung, wo der Größenzuwachs zwischen den Teilstichproben schon eine bedeutsame Minderung der Zufallsschwankungen der Assoziationskoeffizienten mit sich bringen kann. Die ausbildungsspezifischen Alterskohorten schwanken in ihrer Größe zwischen $n=19$ und $n=781$, und ein solcher Stichprobenzuwachs verkleinert Zufallsschwankungen ganz erheblich.
Tabelle 7 Kommunalitäten $\left(r^{2}\right)$ der zweidimensionalen Konfigurationen für ausbildungsspezifische Alterskohorten zu jedem Zeitpunkt mit der Durchschnittskonfiguration aus den Gesamtgruppen

\begin{tabular}{|c|c|c|c|c|c|}
\hline \multirow{2}{*}{\multicolumn{2}{|c|}{$\begin{array}{c}\text { Kohorte, } \\
\text { Jahr }\end{array}$}} & \multicolumn{2}{|c|}{ Hauptschulabschluß } & \multicolumn{2}{|c|}{$\begin{array}{c}\text { Mehr als } \\
\text { Hauptschulabschluß }\end{array}$} \\
\hline & & $(n)$ & $r^{2}$ & (n) & $r^{2}$ \\
\hline \multirow[t]{3}{*}{ A } & 1958 & $(86)$ & $.18^{\star \star \star}$ & (19) & $.55^{\star \star}$ \\
\hline & 1973 & (607) & $.48^{* *}$ & (264) & $.33^{\star \star \star}$ \\
\hline & 1979 & (217) & $.66^{*}$ & (172) & $.50^{\star \star}$ \\
\hline \multirow[t]{3}{*}{ B } & 1958 & (367) & .89 & (85) & $.46^{* *}$ \\
\hline & 1973 & $(781)$ & .88 & (264) & $.46^{\star \star}$ \\
\hline & 1979 & (363) & .77 & $(146)$ & $.26^{* \star *}$ \\
\hline \multirow[t]{3}{*}{ C } & 1958 & $(454)$ & $.33^{\star \star *}$ & $(118)$ & $.71^{*}$ \\
\hline & 1973 & (673) & .86 & (198) & .85 \\
\hline & 1979 & (292) & .80 & (117) & $.68^{*}$ \\
\hline \multirow[t]{3}{*}{ D } & 1958 & $(381)$ & $.65^{\star}$ & $(70)$ & .92 \\
\hline & 1973 & (503) & $.71^{\star}$ & (136) & $.57^{\star \star}$ \\
\hline & 1979 & $(136)$ & $.33^{\star \star \star}$ & (34) & $.29^{\star \star \star}$ \\
\hline
\end{tabular}

\begin{tabular}{|c|c|c|}
\hline Ver & eilung für $r^{2}$ & \\
\hline$\star \star \star \star$ & $.18 \leqslant r^{2} \leqslant .33$ & \\
\hline$\star \star$ & $.43 \leqslant r^{2} \leqslant .57$ & \\
\hline & $.65 \leqslant r^{2} \leqslant .71$ & \\
\hline & $.77 \leqslant r^{2} \leqslant .92$ & \\
\hline
\end{tabular}

einmal an unabhängigen und nicht durch natürliche $\mathrm{Ab}$ - und Zugänge verzerrten Populationen. Als zweites fällt auf, daß in den Stichproben, die durch die Grenzen des Altersspektrums unterrepräsentiert sein müssen, die Kommunalität am geringsten ist: in der Kohorte A 1958, wo im Gegensatz zu den beiden späteren Zeitpunkten nur vier Geburtsjahrgänge berücksichtigt werden, und in der Kohorte D 1973 und 1979, wo die Kohorte nur noch über 64- bzw. über 70jährige umfaßt. In diesen Stichproben kann sowohl die relativ geringe Stichprobengröße der in die multidimensionale Skalierung eingegebenen Assoziationskoeffizienten Zufallsfehler produziert als auch eine Selektion durch Geburtsjahrgang und durch Mortalität systematisch die Beziehungsstruktur verzerrt haben. Wenn man diese beiden Besonderheiten berücksichtigt, so stimmen die mittleren Kohorten vor allem mit Hauptschulausbildung gut mit der Durchschnittskonfiguration überein. Abweichen- 
de und übereinstimmende Kohorten verteilen sich also nach einem groben Muster: Abweichende Kohorten finden sich eher in der besser ausgebildeten und extremen, übereinstimmende Kohorten eher in den weniger ausgebildeten und mittleren Kohorten.

Wie läßt sich nun der Bedeutungswandel der schulischen Zielwerte in den ausbildungsspezifischen Alterskohorten zwischen 1958 und 1979 beschreiben? Man könnte wieder - wie bei der Gruppe mit mehr als Hauptschulabschluß (Abb. 2) - die Entwicklung der Kohorten grafisch verfolgen; ganz unabhängig aber davon, da $\beta$ hierzu insgesamt 8 Abbildungen erforderlich wären, sind diese Abbildungen wegen der oft starken Wandlungen sehr unübersichtlich. Um zu einer übersichtlichen und zusammenfassenden Darstellung zu kommen, habe ich daher für jeden schulischen Zielwert die euklidischen Distanzen zwischen der Durchschnittskonfiguration und der Konfiguration für jede Kohorte zu jedem Zeitpunkt errechnet. Diese Distanz gibt an, wieweit das Verständnis eines bestimmten schulischen Zielwerts in einer bestimmten ausbildungsspezifischen Alterskohorte zu einem bestimmten Zeitpunkt vom allgemeinen Verständnis in den Gesamtgruppen entfernt ist. ${ }^{16}$ Die Distanzen sind in Tab. 8 dargestellt. Die GröBe der Distanzen muß im Vergleich mit dem „Durchmesser" des Circumplexes in Abb. 1 gesehen werden, der ungefähr .70 beträgt. Zur Orientierung sind alle Distanzen über .20 in Tab. 8 hervorgehoben. In Tab. 8 müssen vor allem die Kohorten betrachtet werden, die durch ein abweichendes Verständnis der schulischen Zielwerte charakterisiert sind. Das sind - wie man aus den

${ }^{16}$ Zwei alternative Messungen für diese Entfernung des kohortenspezifischen vom durchschnittlichen Verständnis der Werte wären denkbar. Als erstes hätte man die Vektorgewichtung ohne Ursprungsverschiebung aus PINDIS verwenden können. In dieser Programm-Option werden die Vektoren vom Ursprung der Konfiguration bis zu einem Punkt der Durchschnittskonfiguration in der Richtung des Vektors soweit gestaucht oder gedehnt, bis das Lot von dem entsprechenden Punkt einer anzupassenden Einzelkonfiguration auf diesen Vektor fällt; die VektorGewichte geben also an, wieweit der Vektor eines Punktes der Durchschnittskonfiguration verkleinert oder vergrößert werden muß, um so nahe wie möglich an den entsprechenden Punkt einer Einzelkonfiguration heranzukommen (siehe Borg/Lingoes 1977). Im hier vorliegenden Problem wird durch die Vektorgewichtung ein entscheidender Aspekt der Bewegungen
Kommunalitäten in Tab. 7 sieht - die Kohorten A und $\mathrm{D}$ mit HauptschulabschluB und die Kohorten A, B und D mit mehr als HauptschulabschluB. Ich will prüfen, ob in diesen Kohorten durchgängig bestimmte Werte besonders häufig vom durchschnittlichen Verständnis abweichen und durch einen besonders starken Bedeutungswandel scheinbare Meinungswandlungen produziert haben können.

Besonders häufige Abweichungen vom durchschnittlichen Verständnis zeigen sich als erstes bei „Persönliche Selbständigkeit“ und "Sicheres Selbstbewußtsein“. Zu einem Teil liegen beide Werte zu allen Zeitpunkten weitab vom durchschnittlichen Verständnis - in Kohorte B und D mit mehr als Hauptschulabschluß. Zu einem anderen Teil entfernt sich der eine Wert vom durchschnittlichen Verständnis, während der andere sich ihm annähert - in Kohorte A und D mit Hauptschulabschluß und Kohorte A mit mehr als Hauptschulabschluß. Es sieht so aus, als würden beide Werte im Laufe der Zeit in ihrer Bedeutung ausgetauscht. Offenbar führt die sehr ähnliche Formulierung hier zu Schwankungen des Bedeutungsverständnisses in einzelnen Kohorten: Beide richten sich auf gleiche Bedeutungskomponenten, aber gerade deshalb kann man schwer auf die gleiche Weise zwischen ihnen unterscheiden. Wenn das zutrifft, dann haben die Bedeutungswandlungen dieser beiden Werte wohl kaum den gemessenen Meinungswandel hervorgebracht. Beide Werte haben in der Gesamtgruppe ja ungefähr 10 Prozentpunkte gewonnen, und diese Zunahme ließe sich aus einem tatsächlichen Zuwachs einer gemeinsamen Komponente der Selbst-Orientierung erklä-

der Werte zwischen den drei Zeitpunkten nicht erfaßt: häufig bewegen sich die Werte nämlich gerade in einer Richtung, die zu der Richtung des Vektors der Durchschnittskonfiguration orthogonal ist. Sehr starke Bewegungen erscheinen dann nur als geringfügige Vektorgewichtungen für die Durchschnittskonfiguration. Als zweites hätte man die Summe der Distanzen für einen Wert zwischen den drei Zeitpunkten errechnen können. Dieses Maß hätte zwar die Bewegung eines Wertes über die Zeit erfaßt, sie aber nicht zur Position dieses Wertes in der Durchschnittskonfiguration in Beziehung gesetzt. Ich habe dieses zweite Maß ebenfalls errechnet. Es ergaben sich keine sehr deutlichen Abweichungen zu den hier berichteten Ergebnissen, da der Wert der Durchschnittskonfiguration doch häufig in dêr Nähe der Werte für die drei Zeitpunkte liegt. - Aus diesen Gründen habe ich auf die Darstellung der beiden alternativen Messungen verzichtet. 
Tabelle 8 Distanzen zwischen der Durchschnittskonfiguration und den Konfigurationen der ausbildungsspezifischen Alterskohorten für jeden schulischen Zielwert

\begin{tabular}{|c|c|c|c|c|c|c|c|c|c|c|c|}
\hline \multirow{3}{*}{\multicolumn{2}{|c|}{$\begin{array}{c}\text { Ausbildung, } \\
\text { Kohorte, } \\
\text { Jahr }\end{array}$}} & \multirow{2}{*}{\multicolumn{3}{|c|}{ kognitiv }} & \multicolumn{6}{|c|}{ Schulische Zielwerte } & \multirow{3}{*}{$\begin{array}{l}\text { Durch- } \\
\text { schnitt }\end{array}$} \\
\hline & & & & & & & & ativ & & & \\
\hline & & KENT & WISN & URTE & ORDN & UMGG & $\mathrm{ACHT}$ & PERS & SELB & LEBE & \\
\hline \multicolumn{2}{|c|}{$\begin{array}{l}\text { Hauptschul- } \\
\text { abschluß }\end{array}$} & & & & & & & & & & \\
\hline \multirow[t]{3}{*}{ A } & 1958 & $.45^{\star *}$ & $.25^{*}$ & .10 & .05 & $.49^{* *}$ & .16 & $.39^{*}$ & .08 & $.67^{\star * *}$ & $.29^{*}$ \\
\hline & 1973 & .14 & $.48^{\star \star}$ & .06 & .03 & $.28^{\star}$ & $.27^{*}$ & $.32^{*}$ & $.21^{*}$ & $.25^{\star}$ & $.23^{*}$ \\
\hline & 1979 & .15 & .09 & .06 & $.22^{*}$ & .07 & $.41^{* *}$ & .17 & $.27^{*}$ & .11 & .17 \\
\hline \multirow{3}{*}{ B } & 1958 & .17 & .13 & .05 & .18 & .02 & .13 & .00 & .08 & .05 & .09 \\
\hline & 1973 & .13 & .15 & .02 & .05 & .03 & .04 & $.20^{*}$ & $.20^{\star}$ & .01 & .09 \\
\hline & 1979 & .07 & .04 & .17 & .15 & $.26^{*}$ & .05 & $.34^{*}$ & .08 & .07 & .13 \\
\hline \multirow[t]{3}{*}{$\mathrm{C}$} & 1958 & .07 & .17 & $.24^{\star}$ & $.27^{\star}$ & $.64^{* \star \star}$ & $.22^{*}$ & .04 & $.21^{\star}$ & $.41^{\star \star *}$ & $.25^{*}$ \\
\hline & 1973 & $.20^{*}$ & .11 & .08 & .07 & .10 & $.20^{\star}$ & .05 & .03 & .16 & .11 \\
\hline & 1979 & .03 & .01 & .12 & .18 & $.31^{\star}$ & .17 & .11 & .14 & .06 & .13 \\
\hline \multirow[t]{3}{*}{ D } & 1958 & $.22^{\star}$ & .19 & $.24^{\star}$ & $.27^{*}$ & .13 & .07 & .05 & $.39^{\star}$ & .04 & .18 \\
\hline & 1973 & .13 & .15 & .18 & .01 & .08 & .06 & .05 & .06 & .15 & .14 \\
\hline & 1979 & .17 & .15 & $.23^{*}$ & .02 & $.71^{\star \star \star *}$ & .02 & $.43^{\star *}$ & .19 & .14 & $.23^{*}$ \\
\hline \multicolumn{2}{|c|}{$\begin{array}{l}\text { Mehr als } \\
\text { Hauptschul- } \\
\text { abschluß }\end{array}$} & & & & & & & & & & \\
\hline \multirow[t]{3}{*}{$A$} & 1958 & .16 & $.21^{\star}$ & .08 & .19 & .12 & .19 & $.24^{\star}$ & $.50^{\star \star}$ & $.23^{\star}$ & $.21^{*}$ \\
\hline & 1973 & $.67^{\star \star \star \star}$ & .07 & .01 & .03 & .09 & $.35^{\star}$ & .18 & $.48^{\star \star}$ & .05 & $.21^{*}$ \\
\hline & 1979 & $.29^{\star}$ & .05 & .10 & $.45^{* *}$ & $.20^{*}$ & $.21^{*}$ & $.40^{* *}$ & .14 & .14 & $.22^{*}$ \\
\hline \multirow[t]{3}{*}{ B } & 1958 & $.57^{\star *}$ & .09 & $.28^{*}$ & $.23^{*}$ & $.26^{\star}$ & .16 & .08 & $.21^{*}$ & $.21^{*}$ & $.23^{*}$ \\
\hline & 1973 & $.25^{\star}$ & $.23^{*}$ & .21 & .08 & .14 & .05 & $.31^{\star}$ & $.52^{\star \star}$ & $.30^{*}$ & $.23^{*}$ \\
\hline & 1979 & $.48^{\star \star}$ & .03 & .14 & $.34^{*}$ & $.22^{*}$ & $.30^{*}$ & $.34^{\star}$ & $.54^{\star *}$ & $.25^{\star}$ & $.29^{\star}$ \\
\hline \multirow[t]{3}{*}{ C } & 1958 & .11 & .10 & .14 & .06 & .02 & $.32^{\star}$ & .13 & $.22^{*}$ & $.31^{*}$ & .16 \\
\hline & 1973 & .12 & $.29^{*}$ & .08 & .18 & .05 & .04 & .04 & .09 & .06 & .11 \\
\hline & 1979 & .11 & .01 & .05 & .06 & .19 & $.39^{*}$ & $.21^{\star}$ & $.30^{*}$ & .12 & .16 \\
\hline \multirow[t]{3}{*}{ D } & 1958 & .16 & .08 & .05 & .04 & .14 & .13 & .10 & .03 & .03 & .08 \\
\hline & 1973 & $.53^{\star}$ & $.20^{*}$ & .15 & .05 & .18 & .12 & $.29^{*}$ & .07 & .01 & .18 \\
\hline & 1979 & .05 & $.66^{\star \star *}$ & .08 & $.33^{*}$ & $.23^{*}$ & .18 & .17 & $.49^{\star \star}$ & .10 & $.26^{\star}$ \\
\hline \multicolumn{2}{|c|}{ Durchschnitt } & $.23^{*}$ & .16 & .12 & .15 & $.21^{*}$ & .18 & $.21^{*}$ & $.23^{\star}$ & .16 & \\
\hline \multicolumn{2}{|c|}{$\begin{array}{l}\% \text {-Differenz } \\
1979-1958^{1}\end{array}$} & 7 & 29 & 28 & -29 & -19 & -25 & 9 & 10 & 4 & \\
\hline
\end{tabular}

* Distanz größer als .20

1 Aus Meulemann 1982a: Tab. 8 erste Zeile

** Distanz größer als .40

*** Distanz größer als .60 
ren, die unabhängig von der Wahl eines der beiden, in ihrer Bedeutung flukturierenden Werte ist. Als zweites zeigen „Kenntnisse für den Beruf“, als drittes „Gute Umgangsformen“ besonders häufige Abweichungen von durchschnittlichem Verständnis. Auf der anderen Seite hat „Achtung vor den Mitmenschen" auch in den ausbildungsspezifischen Alterskohorten eine vergleichsweise stabile Bedeutung.

Hängt das Muster von Bedeutungsstabilität und Bedeutungswandel mit dem gemessenen Meinungswandel (Abschnitt 2) zusammen? Am Fuße der Tab. 8 sind die Durchschnitte der Distanzen aus allen 24 Messungen in altersspezifischen Ausbildungskohorten und die Prozentsatzdifferenzen zwischen der Wahl eines Wertes 1979 und 1958 dargestellt. Durch die Bank zeigen genau die Werte den geringsten Bedeutungswandel, bei denen der größte Meinungswandel gemessen wurde. „Vielseitiges Wissen“ und „Eigene Urteilsfähigkeit" gehören zu den stabilen Werten und zeigen mit rund 30 Prozentpunkten Zuwachs die stärkste Veränderung zwischen 1958 und 1979; ebenso gehören „Ordnung und Disziplin“ und „Achtung vor den Mitmenschen" zu den stabilen Werten und nehmen in der Zeit um 29 bzw. 25 Prozentpunkte ab. Auf der anderen Seite zeigen die am wenigsten stabilen Werte - „Kenntnisse für den Beruf", „Persönliche Selbständigkeit" und "Sicheres Selbstbewußtsein" mit 7, 9 und 10 Prozentpunkten $\mathrm{Zu}$ wachs einen relativ schwachen gemessenen Meinungswandel. Allein „Umgang mit den Mitmenschen" verbindet geringe Bedeutungsstabilität mit hohem Meinungswandel, während „Lebensfreude $^{\text {" }}$ weder einen nennenswerten Meinungswandel noch einen nennenswerten Bedeutungswandel aufweist. Insgesamt herrscht also über die Werte der höchste Bedeutungskonsens zwischen Generationen und Bildungsgruppen, für die der stärkste Meinungswandel gemessen wurde.

\section{Meinungs- und Bedeutungswandel: Zusammenfassung und SchluBfolgerungen}

Daß sich Antworthäufigkeiten aus Umfragen in der Zeit verändern, kann auf zwei Aspekte des Wandels hindeuten: auf einen Meinungs- oder einen Bedeutungswandel. Beide Aspekte lassen sich nicht trennen, wenn man nur eine Frage über die Zeit vergleicht. Sobald man aber mehrere Fragen, über deren Beziehungsstruktur Hypothesen vorgegeben sind, zur Verfügung hat, kann man die Beziehung zwischen den erfragten Werten als Indi- kator für ihre Bedeutung ansehen. Auch in der semantischen Theorie (Katz/Foder 1963) wird die Bedeutung eines Wortes ja durch die Beziehung zu anderen Wortbedeutungen dargestellt. Da Beziehungen unabhängig von Häufigkeiten statistisch erfaßt werden können, läßt sich der Meinungswandel vom Bedeutungswandel trennen. Aus einem gemessenen Wandel lassen sich dann Hypothesen über den Bedeutungswandel entwickeln, aber unabhängig vom gemessenen Wandel untersuchen. Als Beispiel einer solchen Forschungsstrategie wurde in diesem Aufsatz ein Inventar schulischer Zielwerte untersucht, das zwischen 1958 und 1979 dreimal erhoben worden war und das sich a priori in kognitive und evaluative und innerhalb der evaluativen wiederum in Konventions- und Autonomie-Werte aufteilen läßt.

Der gemessene Wandel zeigt ein deutliches Ansteigen kognitiver auf Kosten evaluativer schulischer Zielwerte, eine Zunahme von zwei selbstorientierten Werten und einen Rückgang von „Achtung vor den Mitmenschen“. Entsprechend kann man als Bedeutungswandel vermuten, daß die kognitiven Werte näher aneinander gerückt sind, die selbst-orientierten Werte sich den kognitiven Werten angenähert haben und „Achtung vor den Mitmenschen" von Autonomie- zu Konventions-Werten gewandert ist. Tatsächlich aber ist in den Gesamtgruppen die Beziehungsstruktur der 9 schulischen Zielwerte fast vollständig unverändert geblieben. Auch bei einer sehr genauen Betrachtung haben sich die selbst-orientierten Werte nicht den kognitiven Werten angenähert, und „Achtung vor den Mitmenschen" ist nicht von Werten der Autonomie zu Werten der Konvention übergegangen. Die Bedeutung der Werte hat sich also nicht gewandelt, obwohl die Werte sich massiv umgeschichtet haben. Der Wertwandel wurde mit einem konstanten Instrument gemessen; er ist allein ein Meinungswandel.

Auch wenn in der Gesamtbevölkerung die Bedeutung der Werte konstant geblieben ist, kann sie sich in den Teilgruppen gegenläufig entwickelt haben. Vor allem mit dem Bildungsgrad und mit der Generationszugehörigkeit kann die Entwicklung der Bedeutung variieren. Disaggregiert man die Stichproben zunächst nach Bildungsabschlüssen, so zeigt sich in der Gruppe mit Hauptschulabschluß genau die gleiche Entwicklung wie in der gesamten Bevölkerung und in der Gruppe mit mehr als Hauptschulabschluß eine weitgehend gleiche Entwicklung: lediglich „Kenntnisse für den Beruf" verändern hier ihre Bedeutung von einem 
mehr der Lebenspraxis angehörenden zu einem spezifisch schulischen Zielwert. Während also die weniger ausgebildeten Bevölkerungsgruppen die Bedeutungen schulischer Zielwerte beibehalten, weichen die besser ausgebildeten Bevölkerungsgruppen zuerst etwas vom vorherrschenden Bedeutungsverständnis $a b$, um sich dann aber vollständig anzupassen. Die Anpassung des Bedeutungsverständnisses in der besser ausgebildeten Gruppe läßt sich aus der im Laufe der Bildungsexpansion verbreiterten Rekrutierung dieser Gruppe sehr leicht verstehen. Disaggregiert man die Stichproben noch weiter nach ausbildungsspezifischen Alterskohorten, so zeigt sich wiederum, da $\beta$ die weniger ausgebildeten Kohorten besser mit den allgemeinen Wertbedeutungen übereinstimmen als die besser ausgebildeten Kohorten und daß vor allem die durch natürliche $\mathrm{Ab}$ - und Zugänge betroffenen Kohorten von der allgemeinen Wertbedeutung abweichen. Bei genauerer Analyse aber sind die Abweichungen der ausbildungsspezifischen Alterskohorten von der allgemeinen Bedeutung in den schulischen Zielwerten konzentriert, die den geringsten gemessenen Wandel aufweisen. Selbst bei einer Betrachtung extrem disaggregierter Stichproben gilt also, daß die Bedeutung der Werte sich gerade da nicht gewandelt hat, wo der gemessene Wandel am stärksten ist. Auch dort also wurde der Wertwandel an den entscheidenden Stellen mit einem konstanten Instrument gemessen, auch dort ist er Meinungswandel ohne Bedeutungswandel.

Insgesamt stimmen diese Ergebnisse positiv, was die Leistungsfähigkeit des Interviews als eines Instruments zur Analyse kulturellen Wandels betrifft (Allerbeck 1976; Hyman 1972). Sie entkräften empirisch einen theoretisch sehr gewichtigen Einwand: daß ein gemessener Wertwandel lediglich einen Bedeutungswandel darstelle, ohne Wandlungen der Meinungen erfassen zu können. Statt dessen zeigt sich durchweg eine Konstanz der Bedeutungen bei einem Wandel der Meinungen. Dies gilt erstens auf dem hohen Aggregationsniveau ganzer Stichproben für alle Werte. Dies gilt zweitens auch auf dem tieferen Aggregationsniveau von Ausbildungsgruppen und ausbildungsspezifischen Alterskohorten: Zwar gibt es hier in ihrer Bedeutung labilere und stabilere Werte; aber gerade die in ihrer Bedeutung stabilsten Werte unterliegen dem stärksten Meinungswandel. Sicher kann das positive Fazit der Untersuchung nicht darüber hinwegtäuschen, daß die Bedeutungen der Werte nur ex post nach der Erhebung in einer standardisierten Befragung untersucht wurden. Möglicherweise finden viele Bedeutungsverständnisse in der formalisierten Interviewsituation und in der vorgeprägten Formulierung des Fragebogens keinen Ausdruck, und in jedem Falle sind derartige Bedeutungsverständnisse nicht mehr greifbar. Aber auch wenn man die Möglichkeit informeller Nuancen ex ante anerkennen muß, bleibt doch erstaunlich, wie wenig sie sich bei einer formellen Prüfung ex post noch wiederfinden.

\section{Literatur}

Allerbeck, Klaus R., 1976: Demokratisierung und sozialer Wandel in der Bundesrepublik Deutschland. Sekundäranalyse von Umfragen 1953-1974. Opladen: Westdeutscher Verlag.

Borg, I., 1981: Anwendungsorientierte multidimensionale Skalierung. Berlin usw.: Springer

Borg, I./Lingoes, J. C., 1977: Ein direkter Transformationsansatz der multidimensionalen Analyse dreimodaler Datenmatrizen: Theorie und Anwendungen. Zeitschrift für Sozialpsychologie 8: 98-114.

Boyd, Richard W./Hyman, Herbert H., 1975: Survey Research. S. 265-350 in: F. I. Greenstein/N. W. Polsby (Hrsg.), Handbook of Political Science. Vol. 7: Strategies of Inquiry. Reading, Mass.: Addison-Wesley.

Coxon, A. P. M./Jones, C. L./Tagg, S. K., 1981: The MDS(X) Series of Multidimensional Scaling Programs. Inter-University/Research Council Series Report No. 51.

Coxon, A. P. M., 1982: The User's Guide to Multidimensional Scaling. London: Heinemann.

Davis, J. A., 1975: The Log Linear Analysis of Survey Replications. S. 37-74 in: Land/Spilerman 1975.

Duncan, O. D., 1975: Measuring Social Change via Replications of Surveys. S. 75-104 in: Land/Spilerman 1975.

Goodman, Leo A., 1978: Analyzing Qualitative/Categorial Data. London usw.: Addison Wesley.

Hyman, Herbert H., 1972: Secondary Analysis of Sample Surveys: Principles, Procedures and Potentialities. New York etc.: Wiley.

Katz, Jerrold J./Fodor, J. A., 1963: The structure of a semantic theory. Language 39: 170-210.

Land, K. C./Spilerman, S., 1975: Social Indicator Models. New York: Russell Sage.

Langeheine, R., 1980: Approximate norms and significance tests for the Lingoes-Borg Procrustean Individual Differences Scaling (PINDIS). Institut für Pädagogik der Naturwissenschaften an der Universität Kiel. IPN-Arbeitsberichte 39.

Lingoes, J./Borg, I., 1977: Identifying spatial manifolds for interpretation. S. 127-148 in: J. C. Lingoes/E. E. Roskam/I. Borg (Hrsg.), Geometric representations of relational data. Readings in multidimensional scaling. Ann Arbor, Michigan: Mathesis Press. 
Meulemann, Heiner, 1982a: Bildungsexpansion und Wandel der Bildungsvorstellungen zwischen 1958 und 1979: Eine Kohortenanalyse. Zeitschrift für Soziologie 11: 227-253.

Meulemann, Heiner, 1982b: Bildung und Weiterbildung in der Bundesrepublik im Wandel. Sekundäranalysen von Umfragen - ausgewählte Forschungsergebnisse. Zentralarchiv Information 11, Universität zu Köln.

Nowak, S., 1977: The Strategy of Cross-National Research for the Development of Social Theory. S. 3-48 in: Szalai, A./Petrella, R. (Hrsg.): Cross-National Comparative Survey Research. Oxford: Pergamon Press.

Parsons, T./Shils, E. A., 1951: Toward a General Theory of Action. Theoretical Foundations for the Social Sciences. Cambridge, Mass.: Harvard University Press.
Przeworski, A./Teune, H., 1970: The Logic of Comparative Social Inquiry. New York: Wiley-Interscience.

Rabinowitz, George, 1975: An Introduction to Nonmetric Multidimensional Scaling. American Journal of Political Science 19: 343-390.

Roskam, E. E., 1975: Non-metric data analysis: General methodology and techniques. University of Nijmegen, Report 75-MA-13.

Schumann, H./Presser, S., 1981: Questions and Answers in Attitude Surveys. Experiments on Question Form, Wording and Context. New York: Academic Press.

Strzelewicz, W./Raapke, H. D./Schulenberg, W., 1966: Bildung und gesellschaftliches Bewußtsein. Eine mehrstufige soziologische Untersuchung in Westdeutschland. Stuttgart: Enke.

Sudman, S./Bradburn, N. M., 1982: Asking Questions. A Practical Guide to Questionnaire Design. San Francisco: Jossey-Bass. 